\section{Crystal structure of anagyrine perchlorate}

\section{Kambarali K. Turgunov, ${ }^{*}$ Shukhrat B. Rakhimov, Valentina I. Vinogradova and Bakhodir Tashkhodjaev}

S. Yunusov Institute of the Chemistry of Plant Substances, Academy of Sciences of Uzbekistan, Mirzo Ulugbek Str. 77, Tashkent 100170, Uzbekistan. *Correspondence e-mail: kk_turgunov@rambler.ru

Received 15 April 2015; accepted 20 April 2015

Edited by W. T. A. Harrison, University of Aberdeen, Scotland

The title molecular salt, $\mathrm{C}_{15} \mathrm{H}_{21} \mathrm{~N}_{2} \mathrm{O}^{+}$. $\mathrm{ClO}_{4}{ }^{-}$, crystallizes with four cations $(A, B, C$ and $D)$ and four anions in the chiral unit cell (space group $P 2_{1}$ ). The alkaloid was isolated from the aerial parts of Genista Hispanica collected in the Samarkand region of Uzbekistan. Each cation is protonated at the $\mathrm{N}$ atom that bridges the alkaloid rings $C$ and $D$. In each cation, ring $A$ is almost planar and ring $B$ adops a sofa conformation with the methylene group bridging to the $C$ ring as the flap. Rings $C$ and $D$ adopt chair conformations with a cis ring junction in all four cations. In the crystal, $A+B$ and $C+D$ dimeric pairs linked by pairs of $\mathrm{N}-\mathrm{H} \cdots \mathrm{O}$ hydrogen bonds are observed, which generate $R_{2}^{2}(16)$ loops in each case. The dimers are consolidated by weak aromatic $\pi-\pi$ stacking interactions between the $A$ rings [centroid-centroid distances $=3.913$ (3) and 3.915 (3) ̊]].

Keywords: crystal structure; alkaloid; Genista Hispanica; anagyrine; perchlorate; $\mathrm{N}-\mathrm{H}$. . O hydrogen bonds; $\pi-\pi$ stacking interactions.

CCDC reference: 1060546

\section{Related literature}

For the isolation of the title alkaloid, see: Orechoff et al. (1934); Sagen et al. (2002). For NMR spectra of the title alkaloid, see: Sagen et al. (2002). For theoretical studies of anagyrine and the crystal structure of anagyrine hydrochloride monohydrate, see: Galasso et al. (2006). For a related crystal structure, see: Atta-ur-Rahman et al. (1991).

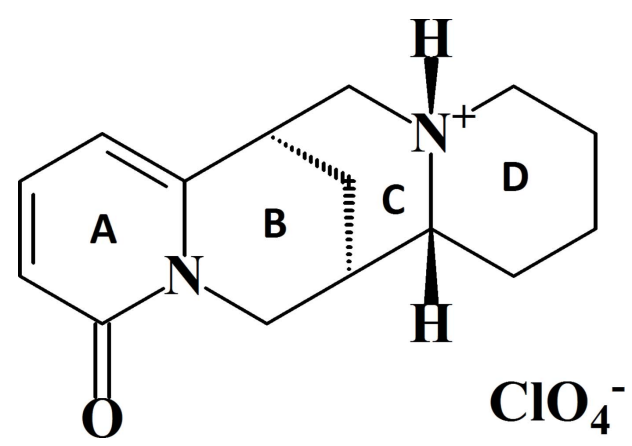

\section{Experimental}

2.1. Crystal data

$\mathrm{C}_{15} \mathrm{H}_{21} \mathrm{~N}_{2} \mathrm{O}^{+} \cdot \mathrm{ClO}_{4}^{-}$

$M_{r}=344.79$

Monoclinic, $P 2$

$a=7.3550$ (3) А

$b=32.982(1) \AA$

$c=12.8849$ (4) $\AA$

$\beta=90.709(3)^{\circ}$

$$
\begin{aligned}
& V=3125.41(19) \AA^{3} \\
& Z=8 \\
& \text { Cu K } \alpha \text { radiation } \\
& \mu=2.42 \mathrm{~mm}^{-1} \\
& T=290 \mathrm{~K} \\
& 0.65 \times 0.15 \times 0.04 \mathrm{~mm}
\end{aligned}
$$

\subsection{Data collection}

Oxford Diffraction Xcalibur Ruby diffractometer

Absorption correction: multi-scan (CrysAlis PRO; Oxford Diffraction, 2009)

$T_{\min }=0.651, T_{\max }=1.000$

\subsection{Refinement}

$R\left[F^{2}>2 \sigma\left(F^{2}\right)\right]=0.064$

$w R\left(F^{2}\right)=0.189$

$S=1.02$

12780 reflections

846 parameters

1 restraint

$\mathrm{H}$ atoms treated by a mixture of independent and constrained refinement

$\Delta \rho_{\max }=0.60{\mathrm{e} \AA^{-3}}^{-3}$

$\Delta \rho_{\min }=-0.37 \mathrm{e}^{-3}$

Absolute structure: Flack $x$ determined using 3107 quotients $\left[\left(I^{+}\right)-\left(I^{-}\right)\right] /\left[\left(I^{+}\right)+\left(I^{-}\right)\right]$(Parsons et al., 2013)

Absolute structure parameter: $-0.024(12)$

Table 1

Hydrogen-bond geometry $\left(\AA{ }^{\circ}\right)$.

\begin{tabular}{lllll}
\hline$D-\mathrm{H} \cdots A$ & $D-\mathrm{H}$ & $\mathrm{H} \cdots A$ & $D \cdots A$ & $D-\mathrm{H} \cdots A$ \\
\hline $\mathrm{N} 2 A-\mathrm{H} 2 A N \cdots \mathrm{O} 1 B$ & $1.03(5)$ & $1.91(6)$ & $2.741(6)$ & $136(5)$ \\
$\mathrm{N} 2 B-\mathrm{H} 2 C N \cdots \mathrm{O} 1 A$ & $0.77(7)$ & $2.00(6)$ & $2.742(5)$ & $163(6)$ \\
$\mathrm{N} 2 C-\mathrm{H} 2 E N \cdots \mathrm{O} 1 D^{\mathrm{i}}$ & $0.90(9)$ & $2.00(9)$ & $2.735(6)$ & $138(8)$ \\
$\mathrm{N} 2 D-\mathrm{H} 2 G N \cdots \mathrm{O} 1 C^{\mathrm{ii}}$ & $1.05(5)$ & $1.74(5)$ & $2.754(5)$ & $159(5)$ \\
\hline
\end{tabular}

Symmetry codes: (i) $x+1, y, z$; (ii) $x-1, y, z$.

Data collection: CrysAlis PRO (Oxford Diffraction, 2009); cell refinement: CrysAlis PRO; data reduction: CrysAlis PRO; program(s) used to solve structure: SHELXS97 (Sheldrick, 2008); program(s) used to refine structure: SHELXL97 (Sheldrick, 2008); molecular graphics: $X P$ in SHELXTL (Sheldrick, 2008); software used to prepare material for publication: publCIF (Westrip, 2010). 


\section{Acknowledgements}

We thank the Academy of Sciences of the Republic of Uzbekistan for supporting this study (grant FA-F7-T185)

Supporting information for this paper is available from the IUCr electronic archives (Reference: HB7409).

\section{References}

Atta-ur-Rahman Pervin, A., Choudhary, M. I., Hasan, N. \& Sener, B. (1991). J. Nat. Prod. 54, 929-935.

Galasso, V., Przybył, A. K., Christov, V., Kovač, B., Asaro, F. \& Zangrando, E. (2006). Chem. Phys. 325, 365-377.

Orechoff, A., Norkina, S. \& Gurewitsch, H. (1934). Berichte, 67, 1394-1398,

Oxford Diffraction (2009). CrysAlis PRO. Oxford Diffraction Ltd, Yarnton, England.

Parsons, S., Flack, H. D. \& Wagner, T. (2013). Acta Cryst. B69, 249-259.

Sagen, A.-L., Gertsch, J., Becker, R., Heilmann, J. \& Sticher, O. (2002). Phytochemistry, 61, 975-978.

Sheldrick, G. M. (2008). Acta Cryst. A64, 112-122.

Westrip, S. P. (2010). J. Appl. Cryst. 43, 920-925. 


\section{supporting information}

Acta Cryst. (2015). E71, o343-0344 [https://doi.org/10.1107/S2056989015007781]

\section{Crystal structure of anagyrine perchlorate}

\section{Kambarali K. Turgunov, Shukhrat B. Rakhimov, Valentina I. Vinogradova and Bakhodir Tashkhodjaev}

\section{S1. Comment}

Quinolizidine alkaloids attracted the attention of researchers due to the structural characteristics and pharmacological activity. We have studied the aerial parts of Genista Hispanica collected in Samarkand region and isolated anagyrine with $\mathrm{R}_{\mathrm{f}} 0.5$ (chloroform-methanol 6:1) along with other alkaloids. Anagyrine a toxic alkaloid found in several species of Lupinus in the western United States. Acute poisoning produces nervousness, depression, loss of muscular control, convulsions, and coma.

Anagyrine perchlorate crystallizes in the monoclinic space group $\mathrm{P} 2{ }_{1}$ with a long unique b-axis of 32.982 (1) $\AA$. The asymmetric unit consist of four protonated anagyrine molecules and four perchlorate anions. The molecular structure of the alkaloid is shown in Fig. 1. The alkaloid molecule consists of four fused rings - planar ring A fused with the sofa ring $\mathrm{B}$, and a twin-chair $\mathrm{C} / \mathrm{D}$ fragment where $\mathrm{C} / \mathrm{D}$ junction is cis. Conformation of all undependent molecules matches. Anagyrine molecule has three asymmetric centers at $\mathrm{C} 6, \mathrm{C} 8, \mathrm{C} 10$ and in addition by protonation of $\mathrm{N} 2$ it becomes as asymmetric senter. Configuration of chiral atoms are C-6R,C-8R,C-10R and N-2S. Crystal structure of thermopsine - a C-10-epimer of anagyrine was investigated by Atta-ur-Rahman et al. (1991).

In the crystal, pairs of hydrogen bonds between protonated $\mathrm{N}$ atom of the base and the carbonyl $\mathrm{O}$ atom link molecules to form two molecular associates (Fig.2, Table 1.). In addition the associates are linked by weak $\pi-\pi$ stacking interactions observed between aromatic rings of molecule [centroid—centroid distance $=3.913$ (3) $\AA$ and 3.915 (3) $\AA$ for undependent molecular pairs ]

\section{S2. Experimental}

\section{S2.1. Synthesis and crystallization}

The powdered air-dried plant material were extracted with $80 \%$ ethanol. After distilling off the alcohol, the residue was acidified with $\mathrm{H}_{2} \mathrm{SO}_{4}$ and washed with chloroform, then the extract was basified with $25 \%$ aqueous ammonia, the sum of alkaloids $(8.81 \mathrm{~g})$ were extracted with chloroform. The resulting sum were dissolved in ethanol and acidified with $\mathrm{HNO}_{3}$ to a weakly acidic medium, precipitated cytisine nitrate crystals $(0.98 \mathrm{~g})$ were separated, and the mother liquor was evaporated. The resulting aqueous residue was basified with $25 \%$ aqueous ammonia and alkaloids was extracted with chloroform. The resulting sum of alkaloids was subjected to column chromatography on silica gel eluting with chloroform-methanol (100: 1) and isolated anagyrine (0.16g). Obtained anagyrine was dissolved in acetone and perchloric acid was added until acidic medium of $\mathrm{pH} 5-6$. Precipitated anagyrine perchlorate crystals were crystallized from methanol with m.p. $315^{\circ} \mathrm{C}$. Colourless prisms were obtained by re-crystallization from water at $50{ }^{\circ} \mathrm{C}$. 


\section{S2.2. Refinement}

Carbon-bound $\mathrm{H}$ atoms were placed geometrically and treated as riding on their parent atoms, with $\mathrm{C}-\mathrm{H}$ distances of $0.93 \AA$ (aromatic), $0.97 \AA$ (methylen), $0.98 \AA$ (tertiary carbon) and were refined with $\mathrm{Uiso}(\mathrm{H})=1.2 \mathrm{Ueq}(\mathrm{C})$ for all hydrogen atoms. N-bound $\mathrm{H}$ atoms involved in the intermolecular hydrogen bonding were found by difference Fourier synthesis and refined isotropically $[\mathrm{N} 2 \mathrm{~A}-\mathrm{H}=1.03(5) \AA, \mathrm{N} 2 \mathrm{~B}-\mathrm{H} 0.77$ (7) $\AA, \mathrm{N} 2 \mathrm{C}-\mathrm{H} 0.90$ (9) $\AA, \mathrm{N} 2 \mathrm{D}-\mathrm{H} 1.05$ (5) A].

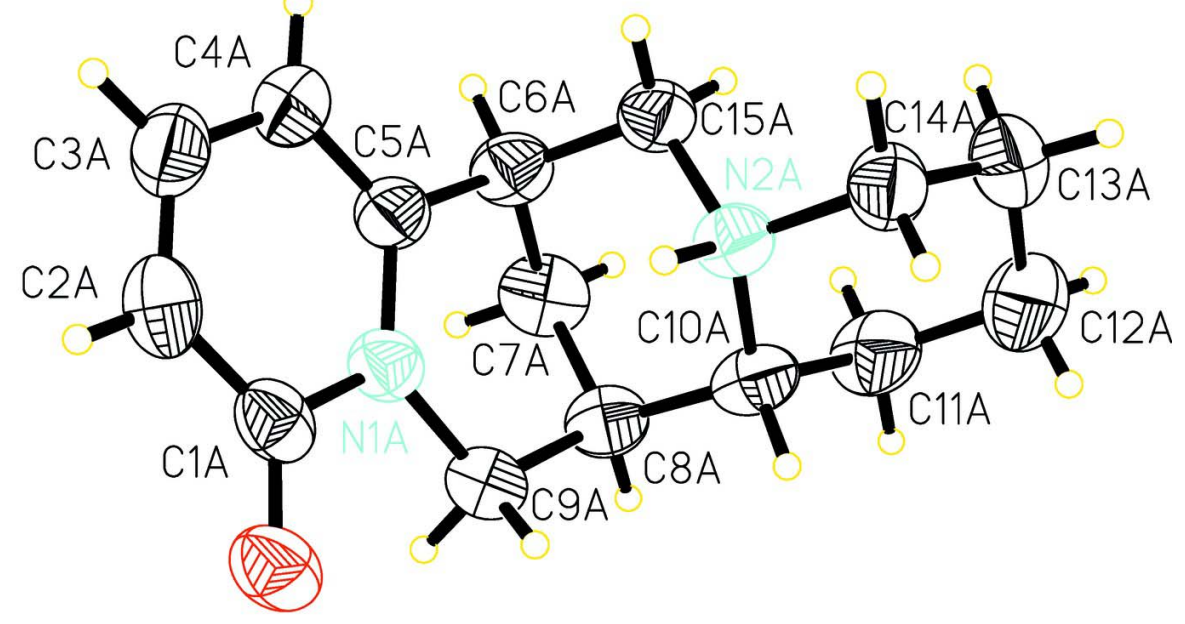

01A

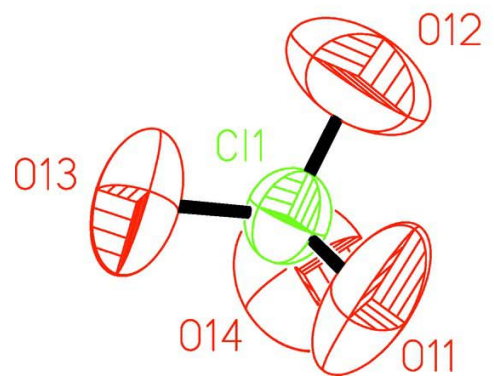

Figure 1

The molecular structure of cation A of the title compound, with displacement ellipsoids drawn at the $50 \%$ probability level. 


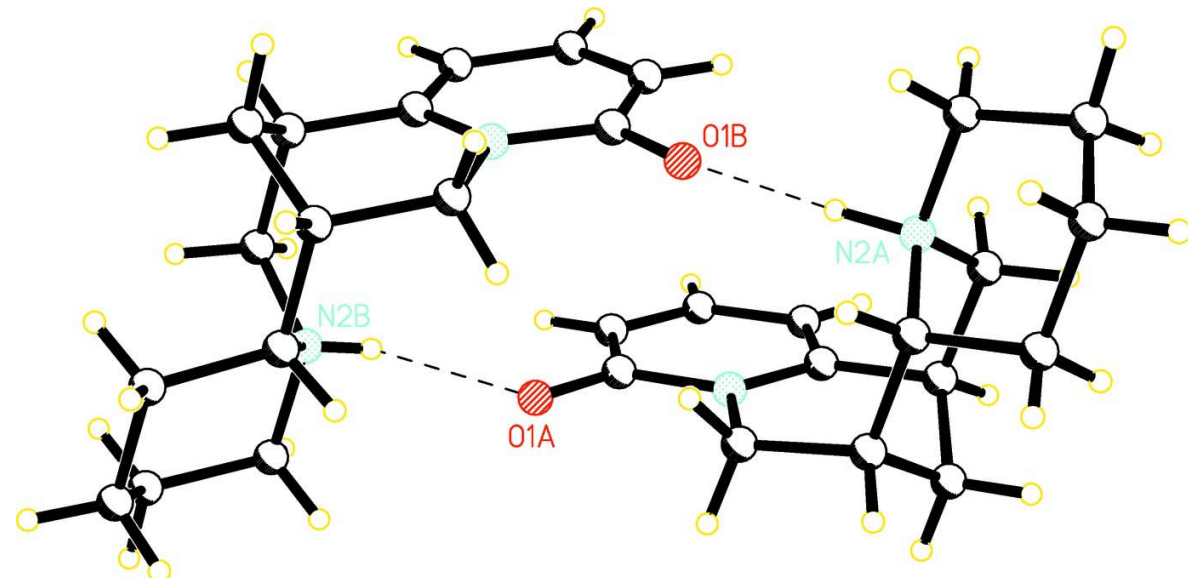

Figure 2

Hydrogen bonding between molecules.

Anagyrine perchlorate

Crystal data

$\mathrm{C}_{15} \mathrm{H}_{21} \mathrm{~N}_{2} \mathrm{O}^{+} \cdot \mathrm{ClO}_{4}^{-}$

$M_{r}=344.79$

Monoclinic, $P 2_{1}$

Hall symbol: $\mathrm{P} 2 \mathrm{yb}$

$a=7.3550$ (3) $\AA$

$b=32.982(1) \AA$

$c=12.8849$ (4) $\AA$

$\beta=90.709(3)^{\circ}$

$V=3125.41(19) \AA^{3}$

$Z=8$

$F(000)=1456$

$D_{\mathrm{x}}=1.465 \mathrm{Mg} \mathrm{m}^{-3}$

Melting point: 588(2) $\mathrm{K}$

$\mathrm{Cu} K \alpha$ radiation, $\lambda=1.54184 \AA$

Cell parameters from 6414 reflections

$\theta=3.7-75.0^{\circ}$

$\mu=2.42 \mathrm{~mm}^{-1}$

$T=290 \mathrm{~K}$

Prism, colourless

$0.65 \times 0.15 \times 0.04 \mathrm{~mm}$

\section{Data collection}

Oxford Diffraction Xcalibur Ruby diffractometer

Radiation source: Enhance $(\mathrm{Cu}) \mathrm{X}$-ray Source

Graphite monochromator

Detector resolution: 10.2576 pixels $\mathrm{mm}^{-1}$

$\omega$ scans

Absorption correction: multi-scan

(CrysAlis PRO; Oxford Diffraction, 2009)

$T_{\min }=0.651, T_{\max }=1.000$

53652 measured reflections

12780 independent reflections

9677 reflections with $I>2 \sigma(I)$

$R_{\text {int }}=0.105$

$\theta_{\max }=77.5^{\circ}, \theta_{\min }=3.4^{\circ}$

$h=-9 \rightarrow 8$

$k=-41 \rightarrow 41$

$l=-16 \rightarrow 16$

Secondary atom site location: difference Fourier map

Hydrogen site location: inferred from neighbouring sites

$\mathrm{H}$ atoms treated by a mixture of independent and constrained refinement

$w=1 /\left[\sigma^{2}\left(F_{\mathrm{o}}^{2}\right)+(0.0663 P)^{2}+0.7864 P\right]$

where $P=\left(F_{\mathrm{o}}{ }^{2}+2 F_{\mathrm{c}}{ }^{2}\right) / 3$

$(\Delta / \sigma)_{\max }=0.005$

$\Delta \rho_{\max }=0.60 \mathrm{e} \AA^{-3}$

$\Delta \rho_{\min }=-0.37 \mathrm{e}^{-3}$ 
Extinction correction: SHELXL,

$\mathrm{Fc}^{*}=\mathrm{kFc}\left[1+0.001 \times \mathrm{Sc}^{2} \lambda^{3} / \sin (2 \theta)\right]^{-1 / 4}$

Extinction coefficient: $0.00064(10)$
Absolute structure: Flack $x$ determined using 3107 quotients $\left[\left(I^{+}\right)-(I)\right] /\left[\left(I^{+}\right)+\left(I^{-}\right)\right]$(Parsons et al., 2013)

Absolute structure parameter: -0.024 (12)

\section{Special details}

Geometry. All e.s.d.'s (except the e.s.d. in the dihedral angle between two l.s. planes) are estimated using the full covariance matrix. The cell e.s.d.'s are taken into account individually in the estimation of e.s.d.'s in distances, angles and torsion angles; correlations between e.s.d.'s in cell parameters are only used when they are defined by crystal symmetry. An approximate (isotropic) treatment of cell e.s.d.'s is used for estimating e.s.d.'s involving l.s. planes.

Refinement. Refinement of $F^{2}$ against ALL reflections. The weighted $R$-factor $w R$ and goodness of fit $S$ are based on $F^{2}$, conventional $R$-factors $R$ are based on $F$, with $F$ set to zero for negative $F^{2}$. The threshold expression of $F^{2}>\sigma\left(F^{2}\right)$ is used only for calculating $R$-factors(gt) etc. and is not relevant to the choice of reflections for refinement. $R$-factors based on $F^{2}$ are statistically about twice as large as those based on $F$, and $R$ - factors based on ALL data will be even larger.

Fractional atomic coordinates and isotropic or equivalent isotropic displacement parameters $\left(\AA^{2}\right)$

\begin{tabular}{|c|c|c|c|c|}
\hline & $x$ & $y$ & $z$ & $U_{\text {iso }} * / U_{\text {eq }}$ \\
\hline O1A & $0.4133(6)$ & $0.31408(11)$ & $0.7883(3)$ & $0.0586(9)$ \\
\hline N1A & $0.5187(5)$ & $0.35415(11)$ & $0.9204(3)$ & $0.0447(8)$ \\
\hline $\mathrm{N} 2 \mathrm{~A}$ & $0.2907(6)$ & $0.38902(11)$ & $1.1098(3)$ & $0.0460(8)$ \\
\hline $\mathrm{C} 1 \mathrm{~A}$ & $0.4817(7)$ & $0.34744(15)$ & $0.8144(4)$ & $0.0485(10)$ \\
\hline $\mathrm{C} 2 \mathrm{~A}$ & $0.5292(7)$ & $0.37879(17)$ & $0.7450(4)$ & $0.0576(12)$ \\
\hline $\mathrm{H} 2 \mathrm{~A}$ & 0.5092 & 0.3752 & 0.6741 & $0.069 *$ \\
\hline $\mathrm{C} 3 \mathrm{~A}$ & $0.6032(8)$ & $0.41377(17)$ & $0.7797(4)$ & $0.0593(12)$ \\
\hline $\mathrm{H} 3 \mathrm{~A}$ & 0.6344 & 0.4340 & 0.7328 & $0.071 *$ \\
\hline $\mathrm{C} 4 \mathrm{~A}$ & $0.6333(8)$ & $0.41978(15)$ & $0.8873(4)$ & $0.0569(12)$ \\
\hline $\mathrm{H} 4 \mathrm{~A}$ & 0.6803 & 0.4443 & 0.9111 & $0.068 *$ \\
\hline $\mathrm{C} 5 \mathrm{~A}$ & 0.5937 (6) & $0.38989(14)$ & $0.9558(4)$ & $0.0468(9)$ \\
\hline C6A & $0.6195(7)$ & $0.39559(14)$ & 1.0709 (4) & $0.0509(10)$ \\
\hline H6A & 0.7237 & 0.4136 & 1.0823 & $0.061^{*}$ \\
\hline C7A & $0.6605(8)$ & $0.35544(16)$ & $1.1242(4)$ & $0.0585(12)$ \\
\hline $\mathrm{H} 7 \mathrm{~A}$ & 0.6815 & 0.3595 & 1.1979 & $0.070 *$ \\
\hline H7B & 0.7678 & 0.3430 & 1.0948 & $0.070^{*}$ \\
\hline C8A & $0.4945(7)$ & $0.32869(14)$ & $1.1059(4)$ & $0.0495(10)$ \\
\hline H8A & 0.5179 & 0.3029 & 1.1412 & $0.059 *$ \\
\hline C9A & $0.4759(7)$ & 0.31962 (13) & $0.9901(4)$ & $0.0500(10)$ \\
\hline H9A & 0.5558 & 0.2972 & 0.9735 & $0.060 *$ \\
\hline H9B & 0.3522 & 0.3109 & 0.9757 & $0.060^{*}$ \\
\hline C10A & $0.3225(8)$ & $0.34697(14)$ & $1.1534(4)$ & $0.0525(11)$ \\
\hline H10A & 0.2194 & 0.3300 & 1.1316 & $0.063^{*}$ \\
\hline C11A & $0.3270(10)$ & $0.34759(18)$ & $1.2714(4)$ & $0.0660(14)$ \\
\hline H11A & 0.4344 & 0.3621 & 1.2953 & $0.079^{*}$ \\
\hline H11B & 0.3345 & 0.3200 & 1.2973 & $0.079 *$ \\
\hline $\mathrm{C} 12 \mathrm{~A}$ & $0.1551(12)$ & $0.3683(2)$ & $1.3153(5)$ & $0.0811(19)$ \\
\hline H12A & 0.0493 & 0.3519 & 1.2989 & $0.097 *$ \\
\hline H12B & 0.1663 & 0.3701 & 1.3903 & $0.097^{*}$ \\
\hline $\mathrm{C} 13 \mathrm{~A}$ & $0.1284(10)$ & 0.41028 (19) & $1.2713(4)$ & 0.0677 (14) \\
\hline H13A & 0.0151 & 0.4215 & 1.2962 & $0.081 *$ \\
\hline
\end{tabular}




\begin{tabular}{|c|c|c|c|c|}
\hline H13B & 0.2268 & 0.4277 & 1.2946 & $0.081^{*}$ \\
\hline C14A & $0.1239(7)$ & $0.40890(16)$ & $1.1549(4)$ & $0.0548(11)$ \\
\hline H14A & 0.1141 & 0.4363 & 1.1283 & $0.066^{*}$ \\
\hline H14B & 0.0167 & 0.3941 & 1.1321 & $0.066^{*}$ \\
\hline $\mathrm{C} 15 \mathrm{~A}$ & $0.4529(7)$ & $0.41532(14)$ & $1.1185(3)$ & $0.0479(10)$ \\
\hline H15A & 0.4777 & 0.4211 & 1.1911 & $0.057^{*}$ \\
\hline H15B & 0.4285 & 0.4409 & 1.0836 & $0.057^{*}$ \\
\hline O1B & $0.0848(6)$ & $0.36310(12)$ & $0.9439(3)$ & $0.0591(9)$ \\
\hline N1B & $0.0180(6)$ & $0.34775(12)$ & 0.7754 & $0.0464(8)$ \\
\hline N2B & $0.2143(5)$ & $0.28075(11)$ & 0.6285 & $0.0428(8)$ \\
\hline C1B & $0.0737(7)$ & $0.37526(15)$ & $0.8519(4)$ & $0.0498(10)$ \\
\hline $\mathrm{C} 2 \mathrm{~B}$ & $0.1114(8)$ & $0.41506(17)$ & $0.8180(5)$ & $0.0633(13)$ \\
\hline $\mathrm{H} 2 \mathrm{C}$ & 0.1467 & 0.4345 & 0.8664 & $0.076^{*}$ \\
\hline $\mathrm{C} 3 \mathrm{~B}$ & $0.0975(9)$ & $0.42544(18)$ & $0.7174(5)$ & $0.0682(14)$ \\
\hline $\mathrm{H} 3 \mathrm{C}$ & 0.1227 & 0.4519 & 0.6974 & $0.082^{*}$ \\
\hline C4B & $0.0450(9)$ & $0.39665(17)$ & $0.6415(5)$ & $0.0648(14)$ \\
\hline $\mathrm{H} 4 \mathrm{C}$ & 0.0387 & 0.4038 & 0.5717 & $0.078^{*}$ \\
\hline C5B & $0.0042(8)$ & $0.35841(16)$ & $0.6721(4)$ & $0.0538(11)$ \\
\hline C6B & $-0.0512(8)$ & $0.32671(17)$ & $0.5943(4)$ & $0.0559(12)$ \\
\hline $\mathrm{H} 6 \mathrm{C}$ & -0.1171 & 0.3400 & 0.5372 & $0.067^{*}$ \\
\hline C7B & $-0.1758(8)$ & $0.2952(2)$ & $0.6424(4)$ & $0.0640(14)$ \\
\hline $\mathrm{H} 7 \mathrm{C}$ & -0.2846 & 0.3081 & 0.6686 & $0.077^{*}$ \\
\hline H7D & -0.2117 & 0.2752 & 0.5910 & $0.077^{*}$ \\
\hline C8B & $-0.0701(7)$ & $0.27531(16)$ & $0.7304(4)$ & $0.0546(11)$ \\
\hline $\mathrm{H} 8 \mathrm{C}$ & -0.1510 & 0.2552 & 0.7614 & $0.066^{*}$ \\
\hline C9B & $-0.0252(7)$ & $0.30642(15)$ & $0.8148(4)$ & $0.0501(10)$ \\
\hline $\mathrm{H} 9 \mathrm{C}$ & -0.1278 & 0.3084 & 0.8611 & $0.060^{*}$ \\
\hline H9D & 0.0780 & 0.2966 & 0.8552 & $0.060^{*}$ \\
\hline $\mathrm{C} 10 \mathrm{~B}$ & $0.0950(7)$ & $0.25221(14)$ & $0.6903(4)$ & $0.0495(10)$ \\
\hline $\mathrm{H} 10 \mathrm{C}$ & 0.1657 & 0.2427 & 0.7505 & $0.059 *$ \\
\hline $\mathrm{C} 11 \mathrm{~B}$ & $0.0438(9)$ & $0.21524(18)$ & $0.6244(5)$ & $0.0679(15)$ \\
\hline $\mathrm{H} 11 \mathrm{C}$ & -0.0204 & 0.1959 & 0.6673 & $0.082 *$ \\
\hline H11D & -0.0384 & 0.2237 & 0.5691 & $0.082 *$ \\
\hline $\mathrm{C} 12 \mathrm{~B}$ & $0.2083(10)$ & $0.19436(17)$ & $0.5769(5)$ & $0.0711(16)$ \\
\hline $\mathrm{H} 12 \mathrm{C}$ & 0.2831 & 0.1827 & 0.6318 & $0.085^{*}$ \\
\hline H12D & 0.1673 & 0.1725 & 0.5321 & $0.085^{*}$ \\
\hline C13B & $0.3193(9)$ & $0.22340(17)$ & $0.5158(5)$ & $0.0653(14)$ \\
\hline $\mathrm{H} 13 \mathrm{C}$ & 0.2485 & 0.2332 & 0.4570 & $0.078^{*}$ \\
\hline H13D & 0.4260 & 0.2097 & 0.4896 & $0.078^{*}$ \\
\hline C14B & $0.3771(7)$ & $0.25872(16)$ & $0.5831(4)$ & $0.0561(11)$ \\
\hline $\mathrm{H} 14 \mathrm{C}$ & 0.4473 & 0.2776 & 0.5420 & $0.067^{*}$ \\
\hline H14D & 0.4546 & 0.2489 & 0.6391 & $0.067 *$ \\
\hline $\mathrm{C} 15 \mathrm{~B}$ & $0.1168(8)$ & $0.30531(15)$ & 0.5495 & $0.0543(11)$ \\
\hline $\mathrm{H} 15 \mathrm{C}$ & 0.0787 & 0.2879 & 0.4927 & $0.065^{*}$ \\
\hline H15D & 0.1991 & 0.3255 & 0.5221 & $0.065^{*}$ \\
\hline $\mathrm{O} 1 \mathrm{C}$ & $0.8812(5)$ & $0.12630(11)$ & 0.2909 & $0.0560(8)$ \\
\hline $\mathrm{N} 1 \mathrm{C}$ & $0.7799(5)$ & $0.08624(11)$ & $0.4217(3)$ & $0.0433(7)$ \\
\hline $\mathrm{N} 2 \mathrm{C}$ & $1.0207(6)$ & $0.05052(11)$ & $0.6095(3)$ & $0.0456(8)$ \\
\hline
\end{tabular}




\begin{tabular}{|c|c|c|c|c|}
\hline $\mathrm{C} 1 \mathrm{C}$ & $0.8095(6)$ & 0.09375 (14) & $0.3167(3)$ & $0.0453(9)$ \\
\hline $\mathrm{C} 2 \mathrm{C}$ & $0.7495(7)$ & $0.06292(16)$ & $0.2460(4)$ & $0.0514(10)$ \\
\hline $\mathrm{H} 2 \mathrm{E}$ & 0.7659 & 0.0666 & 0.1752 & $0.062 *$ \\
\hline $\mathrm{C} 3 \mathrm{C}$ & $0.6694(8)$ & $0.02861(16)$ & $0.2800(4)$ & $0.0574(12)$ \\
\hline $\mathrm{H} 3 \mathrm{E}$ & 0.6292 & 0.0093 & 0.2324 & $0.069^{*}$ \\
\hline $\mathrm{C} 4 \mathrm{C}$ & $0.6467(8)$ & $0.02195(15)$ & $0.3858(4)$ & $0.0550(11)$ \\
\hline $\mathrm{H} 4 \mathrm{E}$ & 0.5940 & -0.0020 & 0.4085 & $0.066^{*}$ \\
\hline $\mathrm{C} 5 \mathrm{C}$ & $0.7012(6)$ & $0.05031(13)$ & $0.4561(4)$ & $0.0449(9)$ \\
\hline $\mathrm{C} 6 \mathrm{C}$ & $0.6872(7)$ & $0.04424(14)$ & $0.5715(4)$ & $0.0498(10)$ \\
\hline H6E & 0.5835 & 0.0263 & 0.5838 & $0.060 *$ \\
\hline $\mathrm{C} 7 \mathrm{C}$ & $0.6506(8)$ & $0.08412(16)$ & $0.6271(4)$ & $0.0561(11)$ \\
\hline $\mathrm{H} 7 \mathrm{E}$ & 0.5412 & 0.0968 & 0.5996 & $0.067^{*}$ \\
\hline $\mathrm{H} 7 \mathrm{~F}$ & 0.6354 & 0.0795 & 0.7009 & $0.067^{*}$ \\
\hline $\mathrm{C} 8 \mathrm{C}$ & $0.8144(8)$ & $0.11066(13)$ & $0.6082(4)$ & $0.0508(11)$ \\
\hline $\mathrm{H} 8 \mathrm{E}$ & 0.7927 & 0.1364 & 0.6441 & $0.061 *$ \\
\hline C9C & $0.8253(8)$ & $0.12038(13)$ & $0.4926(4)$ & $0.0500(10)$ \\
\hline H9E & 0.7433 & 0.1427 & 0.4774 & $0.060 *$ \\
\hline $\mathrm{H} 9 \mathrm{~F}$ & 0.9476 & 0.1295 & 0.4778 & $0.060^{*}$ \\
\hline $\mathrm{C} 10 \mathrm{C}$ & $0.9917(7)$ & $0.09288(14)$ & $0.6542(4)$ & $0.0509(10)$ \\
\hline $\mathrm{H} 10 \mathrm{E}$ & 1.0924 & 0.1100 & 0.6312 & $0.061^{*}$ \\
\hline $\mathrm{C} 11 \mathrm{C}$ & $0.9978(10)$ & $0.09176(17)$ & $0.7727(4)$ & $0.0647(14)$ \\
\hline H11E & 0.9915 & 0.1193 & 0.7989 & $0.078^{*}$ \\
\hline $\mathrm{H} 11 \mathrm{~F}$ & 0.8920 & 0.0773 & 0.7973 & $0.078 *$ \\
\hline $\mathrm{C} 12 \mathrm{C}$ & $1.1679(11)$ & $0.0716(2)$ & $0.8160(5)$ & $0.0760(17)$ \\
\hline $\mathrm{H} 12 \mathrm{E}$ & 1.2733 & 0.0879 & 0.7989 & $0.091^{*}$ \\
\hline $\mathrm{H} 12 \mathrm{~F}$ & 1.1605 & 0.0701 & 0.8910 & $0.091^{*}$ \\
\hline $\mathrm{C} 13 \mathrm{C}$ & $1.1914(10)$ & $0.0292(2)$ & $0.7725(4)$ & $0.0715(15)$ \\
\hline H13E & 1.0937 & 0.0119 & 0.7962 & $0.086^{*}$ \\
\hline $\mathrm{H} 13 \mathrm{~F}$ & 1.3057 & 0.0178 & 0.7970 & $0.086^{*}$ \\
\hline $\mathrm{C} 14 \mathrm{C}$ & $1.1893(8)$ & $0.03078(18)$ & $0.6545(4)$ & $0.0598(12)$ \\
\hline H14E & 1.2953 & 0.0457 & 0.6316 & $0.072 *$ \\
\hline $\mathrm{H} 14 \mathrm{~F}$ & 1.1983 & 0.0034 & 0.6277 & $0.072 *$ \\
\hline $\mathrm{C} 15 \mathrm{C}$ & $0.8559(7)$ & $0.02379(14)$ & $0.6161(3)$ & $0.0489(10)$ \\
\hline $\mathrm{H} 15 \mathrm{E}$ & 0.8351 & 0.0168 & 0.6881 & $0.059^{*}$ \\
\hline $\mathrm{H} 15 \mathrm{~F}$ & 0.8781 & -0.0012 & 0.5785 & $0.059 *$ \\
\hline O1D & $0.2197(6)$ & $0.07898(11)$ & $0.4471(3)$ & $0.0569(8)$ \\
\hline N1D & $0.2731(5)$ & $0.09373(11)$ & $0.2775(3)$ & $0.0424(7)$ \\
\hline N2D & $0.0690(5)$ & $0.16005(10)$ & $0.1287(3)$ & $0.0403(7)$ \\
\hline C1D & $0.2222(7)$ & $0.06657(14)$ & $0.3553(3)$ & $0.0464(10)$ \\
\hline $\mathrm{C} 2 \mathrm{D}$ & $0.1765(7)$ & $0.02692(15)$ & $0.3226(4)$ & $0.0546(11)$ \\
\hline $\mathrm{H} 2 \mathrm{G}$ & 0.1471 & 0.0074 & 0.3718 & $0.065^{*}$ \\
\hline C3D & $0.1750(8)$ & $0.01683(15)$ & $0.2199(4)$ & $0.0569(12)$ \\
\hline $\mathrm{H} 3 \mathrm{G}$ & 0.1380 & -0.0090 & 0.1996 & $0.068^{*}$ \\
\hline C4D & $0.2279(8)$ & $0.04458(15)$ & $0.1455(4)$ & $0.0540(11)$ \\
\hline $\mathrm{H} 4 \mathrm{G}$ & 0.2302 & 0.0371 & 0.0759 & $0.065^{*}$ \\
\hline C5D & $0.2762(7)$ & $0.08259(14)$ & $0.1743(3)$ & $0.0461(9)$ \\
\hline C6D & $0.3330(7)$ & $0.11426(15)$ & $0.0955(3)$ & $0.0492(10)$ \\
\hline H6G & 0.3962 & 0.1004 & 0.0391 & $0.059 *$ \\
\hline
\end{tabular}




\begin{tabular}{|c|c|c|c|c|}
\hline C7D & $0.4602(7)$ & $0.14484(17)$ & $0.1420(4)$ & $0.0554(12)$ \\
\hline $\mathrm{H} 7 \mathrm{G}$ & 0.4957 & 0.1644 & 0.0900 & $0.066^{*}$ \\
\hline $\mathrm{H} 7 \mathrm{H}$ & 0.5688 & 0.1315 & 0.1685 & $0.066^{*}$ \\
\hline $\mathrm{C} 8 \mathrm{D}$ & $0.3609(7)$ & $0.16602(14)$ & $0.2301(4)$ & $0.0508(10)$ \\
\hline H8G & 0.4437 & 0.1863 & 0.2599 & $0.061 *$ \\
\hline C9D & $0.3199(7)$ & $0.13482(14)$ & $0.3155(3)$ & $0.0480(10)$ \\
\hline H9G & 0.4254 & 0.1328 & 0.3611 & $0.058 *$ \\
\hline $\mathrm{H} 9 \mathrm{H}$ & 0.2198 & 0.1449 & 0.3565 & $0.058 *$ \\
\hline C10D & $0.1933(7)$ & $0.18822(13)$ & $0.1895(3)$ & $0.0474(10)$ \\
\hline $\mathrm{H} 10 \mathrm{G}$ & 0.1259 & 0.1980 & 0.2496 & $0.057^{*}$ \\
\hline C11D & $0.2425(9)$ & $0.22534(15)$ & $0.1232(5)$ & $0.0623(14)$ \\
\hline $\mathrm{H} 11 \mathrm{G}$ & 0.3119 & 0.2443 & 0.1656 & $0.075^{*}$ \\
\hline $\mathrm{H} 11 \mathrm{H}$ & 0.3192 & 0.2167 & 0.0667 & $0.075^{*}$ \\
\hline C12D & $0.0761(9)$ & $0.24698(16)$ & $0.0785(5)$ & $0.0662(14)$ \\
\hline $\mathrm{H} 12 \mathrm{G}$ & 0.0047 & 0.2582 & 0.1344 & $0.079 *$ \\
\hline $\mathrm{H} 12 \mathrm{H}$ & 0.1144 & 0.2692 & 0.0344 & $0.079 *$ \\
\hline C13D & $-0.0384(9)$ & $0.21742(18)$ & $0.0160(5)$ & $0.0664(14)$ \\
\hline $\mathrm{H} 13 \mathrm{G}$ & 0.0306 & 0.2077 & -0.0427 & $0.080 *$ \\
\hline $\mathrm{H} 13 \mathrm{H}$ & -0.1460 & 0.2311 & -0.0106 & $0.080^{*}$ \\
\hline C14D & $-0.0952(7)$ & $0.18134(16)$ & $0.0832(4)$ & $0.0540(11)$ \\
\hline $\mathrm{H} 14 \mathrm{G}$ & -0.1719 & 0.1908 & 0.1389 & $0.065^{*}$ \\
\hline $\mathrm{H} 14 \mathrm{H}$ & -0.1652 & 0.1624 & 0.0414 & $0.065^{*}$ \\
\hline C15D & $0.1650(7)$ & $0.13525(14)$ & $0.0495(3)$ & $0.0498(10)$ \\
\hline $\mathrm{H} 15 \mathrm{G}$ & 0.2014 & 0.1526 & -0.0073 & $0.060 *$ \\
\hline $\mathrm{H} 15 \mathrm{H}$ & 0.0822 & 0.1149 & 0.0218 & $0.060 *$ \\
\hline $\mathrm{Cl1}$ & $0.5283(2)$ & $0.18310(5)$ & $0.83543(11)$ & $0.0683(3)$ \\
\hline O11 & $0.3429(11)$ & $0.1756(3)$ & $0.8283(6)$ & $0.150(4)$ \\
\hline $\mathrm{O} 12$ & $0.5416(16)$ & $0.2170(3)$ & $0.8995(9)$ & $0.181(5)$ \\
\hline $\mathrm{O} 13$ & $0.6026(10)$ & 0.1909 (2) & $0.7395(5)$ & $0.120(2)$ \\
\hline $\mathrm{O} 14$ & $0.6176(15)$ & $0.1521(3)$ & $0.8875(7)$ & $0.161(4)$ \\
\hline $\mathrm{Cl} 2$ & $0.6107(3)$ & $0.42676(4)$ & $0.45172(10)$ & $0.0705(4)$ \\
\hline $\mathrm{O} 21$ & $0.452(2)$ & $0.4201(8)$ & $0.4894(10)$ & $0.355(15)$ \\
\hline $\mathrm{O} 22$ & $0.590(2)$ & $0.4423(4)$ & $0.3594(7)$ & $0.244(8)$ \\
\hline $\mathrm{O} 23$ & $0.670(2)$ & $0.3903(3)$ & $0.4433(15)$ & $0.298(11)$ \\
\hline $\mathrm{O} 24$ & $0.7186(14)$ & $0.4502(2)$ & $0.5180(6)$ & 0.153 \\
\hline $\mathrm{Cl} 3$ & $0.7597(2)$ & $0.25649(4)$ & $0.33650(10)$ & $0.0654(3)$ \\
\hline $\mathrm{O} 31$ & $0.6728(15)$ & $0.2873(3)$ & $0.3883(7)$ & $0.167(4)$ \\
\hline $\mathrm{O} 32$ & $0.7512(19)$ & $0.2224(3)$ & $0.3989(9)$ & $0.195(5)$ \\
\hline $\mathrm{O} 33$ & $0.6803(8)$ & 0.24878 (19) & 0.2401 (4) & 0.1007 (18) \\
\hline $\mathrm{O} 34$ & $0.9439(11)$ & $0.2649(4)$ & $0.3267(6)$ & $0.182(5)$ \\
\hline $\mathrm{Cl} 4$ & $0.7284(3)$ & $0.02077(4)$ & $0.96609(10)$ & $0.0700(4)$ \\
\hline O41 & $0.8759(18)$ & $-0.0026(3)$ & $1.0007(9)$ & $0.196(6)$ \\
\hline O42 & $0.5883(17)$ & $0.0062(4)$ & $1.0232(7)$ & $0.198(6)$ \\
\hline $\mathrm{O} 43$ & $0.7115(10)$ & $0.01230(17)$ & $0.8606(3)$ & $0.0973(18)$ \\
\hline O44 & $0.7781(14)$ & $0.06101(17)$ & $0.9878(4)$ & $0.135(3)$ \\
\hline $\mathrm{H} 2 \mathrm{AN}$ & $0.259(8)$ & $0.3892(17)$ & $1.032(4)$ & $0.051(14)^{*}$ \\
\hline $\mathrm{H} 2 \mathrm{CN}$ & $0.264(9)$ & $0.2945(18)$ & $0.668(5)$ & $0.052(15)^{*}$ \\
\hline H2EN & $1.034(13)$ & $0.055(3)$ & $0.541(7)$ & $0.11(3)^{*}$ \\
\hline
\end{tabular}




$\begin{array}{lllll}\text { H2GN } & 0.015(7) & 0.1414(16) & 0.187(4) & 0.043(13)^{*}\end{array}$

Atomic displacement parameters $\left(\AA^{2}\right)$

\begin{tabular}{|c|c|c|c|c|c|c|}
\hline & $U^{11}$ & $U^{22}$ & $U^{33}$ & $U^{12}$ & $U^{13}$ & $U^{23}$ \\
\hline O1A & $0.060(2)$ & 0.0617 (19) & 0.0538 (19) & $-0.0031(16)$ & $-0.0063(16)$ & $-0.0159(15)$ \\
\hline N1A & $0.040(2)$ & $0.0453(17)$ & 0.0491 (19) & $-0.0026(15)$ & $0.0007(15)$ & $-0.0076(14)$ \\
\hline $\mathrm{N} 2 \mathrm{~A}$ & $0.050(2)$ & $0.0452(17)$ & $0.0423(18)$ & $-0.0046(16)$ & $-0.0050(16)$ & $0.0012(14)$ \\
\hline C1A & $0.038(2)$ & $0.055(2)$ & $0.053(2)$ & 0.0049 (19) & $-0.0001(18)$ & $-0.0126(19)$ \\
\hline $\mathrm{C} 2 \mathrm{~A}$ & $0.048(3)$ & $0.074(3)$ & $0.051(3)$ & $0.010(2)$ & 0.007 (2) & $-0.002(2)$ \\
\hline C3A & $0.058(3)$ & $0.062(3)$ & $0.059(3)$ & $0.001(2)$ & $0.015(2)$ & $0.006(2)$ \\
\hline $\mathrm{C} 4 \mathrm{~A}$ & $0.058(3)$ & $0.055(3)$ & $0.058(3)$ & $-0.010(2)$ & $0.013(2)$ & $-0.002(2)$ \\
\hline C5A & $0.040(2)$ & $0.045(2)$ & $0.055(2)$ & $-0.0056(18)$ & $0.0040(18)$ & $-0.0063(18)$ \\
\hline C6A & $0.045(3)$ & $0.052(2)$ & $0.056(3)$ & $-0.0108(19)$ & $-0.0066(19)$ & -0.0060 (19) \\
\hline C7A & $0.053(3)$ & $0.062(3)$ & $0.061(3)$ & $0.003(2)$ & $-0.017(2)$ & $-0.001(2)$ \\
\hline C8A & $0.051(3)$ & $0.045(2)$ & $0.053(2)$ & $-0.0014(18)$ & $-0.008(2)$ & $0.0045(17)$ \\
\hline C9A & $0.054(3)$ & $0.044(2)$ & $0.052(2)$ & $0.0011(19)$ & $-0.002(2)$ & $-0.0052(17)$ \\
\hline C10A & $0.061(3)$ & $0.046(2)$ & $0.051(2)$ & $-0.014(2)$ & $-0.008(2)$ & $0.0050(18)$ \\
\hline C11A & $0.090(4)$ & $0.062(3)$ & $0.045(3)$ & -0.007 & $-0.003(3)$ & $0.014(2)$ \\
\hline C12A & $0.107(6)$ & $0.081(4)$ & $0.055(3)$ & $-0.011(4)$ & $0.023(3)$ & $0.011(3)$ \\
\hline C13A & $0.076(4)$ & $0.071(3)$ & $0.057(3)$ & $0.003(3)$ & $0.016(3)$ & $-0.007(2)$ \\
\hline C14A & $0.054(3)$ & $0.059(3)$ & $0.051(2)$ & $-0.003(2)$ & $0.006(2)$ & $-0.001(2)$ \\
\hline $\mathrm{C} 15 \mathrm{~A}$ & $0.051(3)$ & $0.047(2)$ & $0.045(2)$ & $-0.0101(19)$ & $-0.0018(18)$ & $-0.0063(16)$ \\
\hline O1B & $0.061(2)$ & $0.070(2)$ & $0.0461(18)$ & 0.0027 (17) & $-0.0119(15)$ & $-0.0126(15)$ \\
\hline N1B & $0.044(2)$ & 0.0549 (19) & 0.0403 (18) & 0.0049 (16) & $-0.0033(15)$ & $-0.0072(15)$ \\
\hline N2B & $0.045(2)$ & $0.0442(17)$ & $0.0396(17)$ & $-0.0071(15)$ & $-0.0041(15)$ & $-0.0049(14)$ \\
\hline $\mathrm{C} 1 \mathrm{~B}$ & $0.039(2)$ & $0.060(2)$ & $0.050(2)$ & 0.0102 (19) & $-0.0046(18)$ & -0.0109 (19) \\
\hline $\mathrm{C} 2 \mathrm{~B}$ & $0.053(3)$ & $0.055(3)$ & $0.081(4)$ & $0.003(2)$ & -0.001 & $-0.018(2)$ \\
\hline $\mathrm{C} 3 \mathrm{~B}$ & $0.060(4)$ & $0.056(3)$ & 0.088 (4) & $0.005(2)$ & $0.011(3)$ & $0.009(3)$ \\
\hline C4B & $0.072(4)$ & $0.064(3)$ & $0.058(3)$ & $0.020(3)$ & $0.001(3)$ & $0.007(2)$ \\
\hline C5B & $0.056(3)$ & $0.063(3)$ & $0.042(2)$ & $0.011(2)$ & $-0.002(2)$ & $0.0016(19)$ \\
\hline C6B & $0.056(3)$ & $0.071(3)$ & $0.041(2)$ & $0.013(2)$ & $-0.013(2)$ & $-0.005(2)$ \\
\hline C7B & 0.039 (3) & $0.090(4)$ & $0.063(3)$ & $-0.001(2)$ & $-0.001(2)$ & $-0.027(3)$ \\
\hline $\mathrm{C} 8 \mathrm{~B}$ & $0.052(3)$ & $0.062(3)$ & $0.051(2)$ & $-0.018(2)$ & 0.007 (2) & $-0.010(2)$ \\
\hline C9B & $0.050(3)$ & $0.060(3)$ & $0.040(2)$ & $-0.006(2)$ & $0.0037(18)$ & $-0.0037(18)$ \\
\hline C10B & $0.053(3)$ & 0.047 (2) & 0.049 (2) & -0.0091 (19) & 0.0043 (19) & $-0.0035(17)$ \\
\hline C11B & $0.067(4)$ & $0.062(3)$ & $0.076(4)$ & $-0.025(3)$ & $0.008(3)$ & $-0.015(3)$ \\
\hline C12B & $0.083(4)$ & $0.055(3)$ & $0.075(4)$ & -0.005 & $-0.004(3)$ & $-0.026(3)$ \\
\hline C13B & $0.061(3)$ & $0.062(3)$ & $0.073(3)$ & $-0.001(2)$ & $0.003(3)$ & $-0.025(3)$ \\
\hline C14B & $0.050(3)$ & $0.057(3)$ & $0.062(3)$ & $0.006(2)$ & $-0.003(2)$ & $-0.013(2)$ \\
\hline C15B & $0.067(3)$ & $0.061(3)$ & $0.035(2)$ & $0.001(2)$ & $0.002(2)$ & $0.0004(18)$ \\
\hline $\mathrm{O} 1 \mathrm{C}$ & $0.057(2)$ & 0.0607 (19) & $0.0500(17)$ & $-0.0013(16)$ & $0.0074(15)$ & $0.0148(14)$ \\
\hline $\mathrm{N} 1 \mathrm{C}$ & $0.043(2)$ & 0.0449 (17) & $0.0420(18)$ & $-0.0007(15)$ & $0.0033(14)$ & $0.0020(14)$ \\
\hline $\mathrm{N} 2 \mathrm{C}$ & $0.053(2)$ & 0.0494 (19) & $0.0346(17)$ & $-0.0036(16)$ & $0.0057(15)$ & $0.0040(14)$ \\
\hline $\mathrm{C} 1 \mathrm{C}$ & $0.041(2)$ & $0.054(2)$ & $0.041(2)$ & 0.0054 (19) & $-0.0002(17)$ & $0.0071(17)$ \\
\hline $\mathrm{C} 2 \mathrm{C}$ & $0.046(3)$ & $0.067(3)$ & $0.041(2)$ & $0.007(2)$ & $-0.0032(18)$ & $-0.0056(19)$ \\
\hline $\mathrm{C} 3 \mathrm{C}$ & $0.056(3)$ & $0.059(3)$ & $0.056(3)$ & $0.009(2)$ & $-0.010(2)$ & $-0.014(2)$ \\
\hline $\mathrm{C} 4 \mathrm{C}$ & $0.057(3)$ & $0.048(2)$ & $0.061(3)$ & $-0.007(2)$ & $0.000(2)$ & $-0.007(2)$ \\
\hline
\end{tabular}




\begin{tabular}{|c|c|c|c|c|c|c|}
\hline $\mathrm{C} 5 \mathrm{C}$ & $0.036(2)$ & $0.050(2)$ & $0.049(2)$ & $-0.0040(17)$ & $0.0008(17)$ & $0.0022(17)$ \\
\hline $\mathrm{C} 6 \mathrm{C}$ & $0.051(3)$ & $0.048(2)$ & $0.050(2)$ & -0.0107 (19) & $0.013(2)$ & $0.0030(18)$ \\
\hline $\mathrm{C} 7 \mathrm{C}$ & $0.054(3)$ & $0.063(3)$ & $0.052(3)$ & $-0.004(2)$ & $0.014(2)$ & $0.001(2)$ \\
\hline $\mathrm{C} 8 \mathrm{C}$ & $0.061(3)$ & $0.042(2)$ & $0.050(2)$ & $-0.0019(19)$ & $0.011(2)$ & -0.0077 (17) \\
\hline $\mathrm{C} 9 \mathrm{C}$ & $0.059(3)$ & $0.045(2)$ & $0.047(2)$ & $-0.0036(19)$ & $-0.001(2)$ & $0.0022(17)$ \\
\hline $\mathrm{C} 10 \mathrm{C}$ & $0.060(3)$ & $0.050(2)$ & $0.043(2)$ & $-0.011(2)$ & $0.0060(19)$ & $0.0022(17)$ \\
\hline $\mathrm{C} 11 \mathrm{C}$ & $0.092(4)$ & $0.061(3)$ & $0.042(2)$ & $-0.005(3)$ & $0.003(2)$ & $-0.007(2)$ \\
\hline $\mathrm{C} 12 \mathrm{C}$ & $0.089(5)$ & $0.088(4)$ & $0.050(3)$ & $-0.013(4)$ & $-0.013(3)$ & $-0.002(3)$ \\
\hline $\mathrm{C} 13 \mathrm{C}$ & $0.077(4)$ & $0.087(4)$ & $0.050(3)$ & $0.004(3)$ & $-0.004(3)$ & $0.011(3)$ \\
\hline $\mathrm{C} 14 \mathrm{C}$ & $0.057(3)$ & 0.068 & 0.055 & $0.009(2)$ & $0.004(2)$ & $0.011(2)$ \\
\hline $\mathrm{C} 15 \mathrm{C}$ & $0.057(3)$ & $0.046(2)$ & $0.044(2)$ & $-0.004(2)$ & $0.0043(19)$ & $0.0037(17)$ \\
\hline O1D & $0.066(2)$ & $0.0650(19)$ & $0.0397(16)$ & $0.0003(17)$ & $0.0116(15)$ & $0.0094(14)$ \\
\hline N1D & $0.042(2)$ & $0.0485(17)$ & $0.0369(16)$ & $0.0005(15)$ & $0.0040(14)$ & $0.0026(13)$ \\
\hline N2D & $0.0384(19)$ & $0.0408(16)$ & $0.0419(17)$ & -0.0041 & $0.0030(14)$ & $0.0061(13)$ \\
\hline C1D & $0.046(3)$ & $0.053(2)$ & $0.041(2)$ & $0.0062(19)$ & $0.0088(17)$ & $0.0103(17)$ \\
\hline $\mathrm{C} 2 \mathrm{D}$ & $0.051(3)$ & $0.051(2)$ & $0.061(3)$ & $0.004(2)$ & $0.005(2)$ & $0.011(2)$ \\
\hline C3D & $0.062(3)$ & $0.044(2)$ & $0.065(3)$ & $0.006(2)$ & $0.007(2)$ & $0.000(2)$ \\
\hline $\mathrm{C} 4 \mathrm{D}$ & $0.060(3)$ & $0.056(2)$ & $0.045(2)$ & $0.010(2)$ & $0.003(2)$ & $-0.0046(19)$ \\
\hline C5D & $0.045(2)$ & $0.055(2)$ & $0.038(2)$ & 0.0077 (19) & $0.0029(17)$ & $0.0017(17)$ \\
\hline C6D & $0.051(3)$ & $0.060(2)$ & $0.0372(19)$ & $0.002(2)$ & $0.0140(18)$ & $0.0031(17)$ \\
\hline C7D & $0.031(2)$ & 0.075 & 0.059 & $-0.007(2)$ & $0.0002(19)$ & 0.028 \\
\hline $\mathrm{C} 8 \mathrm{D}$ & $0.047(3)$ & $0.053(2)$ & $0.051(2)$ & $-0.0144(19)$ & $-0.0042(19)$ & $0.0082(19)$ \\
\hline C9D & $0.049(3)$ & $0.058(2)$ & $0.037(2)$ & $-0.006(2)$ & $-0.0053(17)$ & $0.0024(17)$ \\
\hline C10D & $0.057(3)$ & $0.042(2)$ & $0.043(2)$ & $-0.0100(19)$ & $0.0025(18)$ & $0.0010(16)$ \\
\hline C11D & $0.073(4)$ & $0.049(2)$ & $0.065(3)$ & $-0.018(2)$ & -0.008 & $0.014(2)$ \\
\hline C12D & $0.080(4)$ & $0.047(2)$ & $0.071(3)$ & $-0.008(2)$ & $0.004(3)$ & $0.016(2)$ \\
\hline C13D & $0.067(4)$ & $0.068(3)$ & $0.065(3)$ & $-0.002(3)$ & $-0.006(3)$ & $0.025(3)$ \\
\hline C14D & $0.047(3)$ & $0.056(2)$ & $0.059(3)$ & $-0.001(2)$ & $0.002(2)$ & $0.018(2)$ \\
\hline C15D & $0.059(3)$ & $0.056(2)$ & $0.0351(19)$ & $0.004(2)$ & $0.0044(18)$ & $0.0027(17)$ \\
\hline $\mathrm{Cl1}$ & $0.0701(9)$ & $0.0747(8)$ & $0.0602(7)$ & $-0.0005(7)$ & $0.0010(6)$ & $0.0107(6)$ \\
\hline O11 & $0.099(5)$ & $0.237(10)$ & $0.114(5)$ & $-0.075(6)$ & $0.002(4)$ & $0.053(6)$ \\
\hline $\mathrm{O} 12$ & $0.197(11)$ & $0.136(7)$ & $0.211(10)$ & $0.006(7)$ & $0.029(8)$ & $-0.090(7)$ \\
\hline $\mathrm{O} 13$ & $0.106(5)$ & $0.149(6)$ & 0.105 & $0.017(4)$ & $0.039(4)$ & 0.050 \\
\hline $\mathrm{O} 14$ & $0.202(10)$ & $0.155(7)$ & $0.124(6)$ & $0.066(7)$ & $-0.028(6)$ & $0.041(5)$ \\
\hline $\mathrm{Cl} 2$ & $0.1003(12)$ & $0.0585(6)$ & $0.0522(6)$ & $0.0064(7)$ & $-0.0139(7)$ & $-0.0059(5)$ \\
\hline $\mathrm{O} 21$ & $0.232(16)$ & $0.68(4)$ & $0.151(10)$ & $-0.22(2)$ & $0.081(10)$ & $-0.093(17)$ \\
\hline $\mathrm{O} 22$ & $0.350(16)$ & $0.269(13)$ & $0.110(6)$ & $-0.210(13)$ & $-0.120(8)$ & $0.074(7)$ \\
\hline $\mathrm{O} 23$ & $0.331(19)$ & $0.117(7)$ & $0.44(2)$ & $0.107(10)$ & $-0.243(18)$ & $-0.132(10)$ \\
\hline $\mathrm{O} 24$ & $0.243(10)$ & $0.091(4)$ & $0.121(5)$ & $-0.004(5)$ & $-0.107(6)$ & $-0.023(4)$ \\
\hline $\mathrm{Cl} 3$ & $0.0663(8)$ & $0.0713(7)$ & $0.0584(7)$ & $0.0034(6)$ & $-0.0049(6)$ & $-0.0103(5)$ \\
\hline $\mathrm{O} 31$ & $0.206(10)$ & $0.174(8)$ & $0.122(6)$ & $0.079(7)$ & $-0.004(6)$ & $-0.070(6)$ \\
\hline $\mathrm{O} 32$ & $0.274(15)$ & $0.119(6)$ & $0.192(10)$ & $0.028(8)$ & $-0.049(9)$ & $0.059(6)$ \\
\hline $\mathrm{O} 33$ & $0.092(4)$ & $0.119(4)$ & $0.090(3)$ & $0.020(3)$ & $-0.037(3)$ & $-0.027(3)$ \\
\hline $\mathrm{O} 34$ & $0.088(5)$ & $0.338(15)$ & $0.119(5)$ & $-0.068(7)$ & $0.002(4)$ & $-0.092(7)$ \\
\hline $\mathrm{Cl} 4$ & $0.1067(12)$ & $0.0591(6)$ & $0.0445(6)$ & $-0.0147(7)$ & $0.0163(6)$ & $-0.0037(5)$ \\
\hline $\mathrm{O} 41$ & $0.260(14)$ & $0.134(7)$ & $0.192(10)$ & $0.046(8)$ & $-0.121(10)$ & $-0.031(7)$ \\
\hline $\mathrm{O} 42$ & $0.242(12)$ & $0.241(11)$ & $0.113(5)$ & $-0.130(10)$ & $0.107(7)$ & $-0.060(6)$ \\
\hline $\mathrm{O} 43$ & $0.148(5)$ & $0.097(3)$ & $0.047(2)$ & -0.033 & $0.012(3)$ & -0.003 \\
\hline
\end{tabular}


Geometric parameters $\left(\AA,{ }^{\circ}\right)$

\begin{tabular}{|c|c|c|c|}
\hline $\mathrm{O} 1 \mathrm{~A}-\mathrm{C} 1 \mathrm{~A}$ & $1.254(6)$ & $\mathrm{C} 1 \mathrm{C}-\mathrm{C} 2 \mathrm{C}$ & $1.432(7)$ \\
\hline $\mathrm{N} 1 \mathrm{~A}-\mathrm{C} 5 \mathrm{~A}$ & $1.377(6)$ & $\mathrm{C} 2 \mathrm{C}-\mathrm{C} 3 \mathrm{C}$ & $1.351(8)$ \\
\hline $\mathrm{N} 1 \mathrm{~A}-\mathrm{C} 1 \mathrm{~A}$ & $1.407(6)$ & $\mathrm{C} 2 \mathrm{C}-\mathrm{H} 2 \mathrm{E}$ & 0.9300 \\
\hline $\mathrm{N} 1 \mathrm{~A}-\mathrm{C} 9 \mathrm{~A}$ & $1.487(6)$ & $\mathrm{C} 3 \mathrm{C}-\mathrm{C} 4 \mathrm{C}$ & $1.393(8)$ \\
\hline $\mathrm{N} 2 \mathrm{~A}-\mathrm{C} 15 \mathrm{~A}$ & $1.478(6)$ & $\mathrm{C} 3 \mathrm{C}-\mathrm{H} 3 \mathrm{E}$ & 0.9300 \\
\hline $\mathrm{N} 2 \mathrm{~A}-\mathrm{C} 14 \mathrm{~A}$ & $1.513(7)$ & $\mathrm{C} 4 \mathrm{C}-\mathrm{C} 5 \mathrm{C}$ & $1.359(7)$ \\
\hline $\mathrm{N} 2 \mathrm{~A}-\mathrm{C} 10 \mathrm{~A}$ & $1.514(6)$ & $\mathrm{C} 4 \mathrm{C}-\mathrm{H} 4 \mathrm{E}$ & 0.9300 \\
\hline $\mathrm{N} 2 \mathrm{~A}-\mathrm{H} 2 \mathrm{AN}$ & $1.03(6)$ & $\mathrm{C} 5 \mathrm{C}-\mathrm{C} 6 \mathrm{C}$ & $1.504(6)$ \\
\hline $\mathrm{C} 1 \mathrm{~A}-\mathrm{C} 2 \mathrm{~A}$ & $1.414(8)$ & $\mathrm{C} 6 \mathrm{C}-\mathrm{C} 15 \mathrm{C}$ & $1.520(7)$ \\
\hline $\mathrm{C} 2 \mathrm{~A}-\mathrm{C} 3 \mathrm{~A}$ & $1.349(8)$ & $\mathrm{C} 6 \mathrm{C}-\mathrm{C} 7 \mathrm{C}$ & $1.524(7)$ \\
\hline $\mathrm{C} 2 \mathrm{~A}-\mathrm{H} 2 \mathrm{~A}$ & 0.9300 & $\mathrm{C} 6 \mathrm{C}-\mathrm{H} 6 \mathrm{E}$ & 0.9800 \\
\hline $\mathrm{C} 3 \mathrm{~A}-\mathrm{C} 4 \mathrm{~A}$ & $1.414(8)$ & $\mathrm{C} 7 \mathrm{C}-\mathrm{C} 8 \mathrm{C}$ & $1.512(7)$ \\
\hline $\mathrm{C} 3 \mathrm{~A}-\mathrm{H} 3 \mathrm{~A}$ & 0.9300 & $\mathrm{C} 7 \mathrm{C}-\mathrm{H} 7 \mathrm{E}$ & 0.9700 \\
\hline $\mathrm{C} 4 \mathrm{~A}-\mathrm{C} 5 \mathrm{~A}$ & $1.357(7)$ & $\mathrm{C} 7 \mathrm{C}-\mathrm{H} 7 \mathrm{~F}$ & 0.9700 \\
\hline $\mathrm{C} 4 \mathrm{~A}-\mathrm{H} 4 \mathrm{~A}$ & 0.9300 & $\mathrm{C} 8 \mathrm{C}-\mathrm{C} 9 \mathrm{C}$ & $1.526(6)$ \\
\hline $\mathrm{C} 5 \mathrm{~A}-\mathrm{C} 6 \mathrm{~A}$ & $1.505(7)$ & $\mathrm{C} 8 \mathrm{C}-\mathrm{C} 10 \mathrm{C}$ & $1.541(8)$ \\
\hline $\mathrm{C} 6 \mathrm{~A}-\mathrm{C} 7 \mathrm{~A}$ & $1.520(7)$ & $\mathrm{C} 8 \mathrm{C}-\mathrm{H} 8 \mathrm{E}$ & 0.9800 \\
\hline $\mathrm{C} 6 \mathrm{~A}-\mathrm{C} 15 \mathrm{~A}$ & $1.523(7)$ & $\mathrm{C} 9 \mathrm{C}-\mathrm{H} 9 \mathrm{E}$ & 0.9700 \\
\hline $\mathrm{C} 6 \mathrm{~A}-\mathrm{H} 6 \mathrm{~A}$ & 0.9800 & $\mathrm{C} 9 \mathrm{C}-\mathrm{H} 9 \mathrm{~F}$ & 0.9700 \\
\hline $\mathrm{C} 7 \mathrm{~A}-\mathrm{C} 8 \mathrm{~A}$ & $1.522(7)$ & $\mathrm{C} 10 \mathrm{C}-\mathrm{C} 11 \mathrm{C}$ & $1.527(6)$ \\
\hline $\mathrm{C} 7 \mathrm{~A}-\mathrm{H} 7 \mathrm{~A}$ & 0.9700 & $\mathrm{C} 10 \mathrm{C}-\mathrm{H} 10 \mathrm{E}$ & 0.9800 \\
\hline $\mathrm{C} 7 \mathrm{~A}-\mathrm{H} 7 \mathrm{~B}$ & 0.9700 & $\mathrm{C} 11 \mathrm{C}-\mathrm{C} 12 \mathrm{C}$ & $1.518(10)$ \\
\hline $\mathrm{C} 8 \mathrm{~A}-\mathrm{C} 9 \mathrm{~A}$ & $1.526(7)$ & $\mathrm{C} 11 \mathrm{C}-\mathrm{H} 11 \mathrm{E}$ & 0.9700 \\
\hline $\mathrm{C} 8 \mathrm{~A}-\mathrm{C} 10 \mathrm{~A}$ & $1.536(8)$ & $\mathrm{C} 11 \mathrm{C}-\mathrm{H} 11 \mathrm{~F}$ & 0.9700 \\
\hline $\mathrm{C} 8 \mathrm{~A}-\mathrm{H} 8 \mathrm{~A}$ & 0.9800 & $\mathrm{C} 12 \mathrm{C}-\mathrm{C} 13 \mathrm{C}$ & $1.517(10)$ \\
\hline C9A-H9A & 0.9700 & $\mathrm{C} 12 \mathrm{C}-\mathrm{H} 12 \mathrm{E}$ & 0.9700 \\
\hline C9A-H9B & 0.9700 & $\mathrm{C} 12 \mathrm{C}-\mathrm{H} 12 \mathrm{~F}$ & 0.9700 \\
\hline $\mathrm{C} 10 \mathrm{~A}-\mathrm{C} 11 \mathrm{~A}$ & $1.521(7)$ & $\mathrm{C} 13 \mathrm{C}-\mathrm{C} 14 \mathrm{C}$ & $1.521(7)$ \\
\hline $\mathrm{C} 10 \mathrm{~A}-\mathrm{H} 10 \mathrm{~A}$ & 0.9800 & $\mathrm{C} 13 \mathrm{C}-\mathrm{H} 13 \mathrm{E}$ & 0.9700 \\
\hline $\mathrm{C} 11 \mathrm{~A}-\mathrm{C} 12 \mathrm{~A}$ & $1.550(10)$ & $\mathrm{C} 13 \mathrm{C}-\mathrm{H} 13 \mathrm{~F}$ & 0.9700 \\
\hline $\mathrm{C} 11 \mathrm{~A}-\mathrm{H} 11 \mathrm{~A}$ & 0.9700 & $\mathrm{C} 14 \mathrm{C}-\mathrm{H} 14 \mathrm{E}$ & 0.9700 \\
\hline $\mathrm{C} 11 \mathrm{~A}-\mathrm{H} 11 \mathrm{~B}$ & 0.9700 & $\mathrm{C} 14 \mathrm{C}-\mathrm{H} 14 \mathrm{~F}$ & 0.9700 \\
\hline $\mathrm{C} 12 \mathrm{~A}-\mathrm{C} 13 \mathrm{~A}$ & 1.507 (9) & $\mathrm{C} 15 \mathrm{C}-\mathrm{H} 15 \mathrm{E}$ & 0.9700 \\
\hline $\mathrm{C} 12 \mathrm{~A}-\mathrm{H} 12 \mathrm{~A}$ & 0.9700 & $\mathrm{C} 15 \mathrm{C}-\mathrm{H} 15 \mathrm{~F}$ & 0.9700 \\
\hline $\mathrm{C} 12 \mathrm{~A}-\mathrm{H} 12 \mathrm{~B}$ & 0.9700 & $\mathrm{O} 1 \mathrm{D}-\mathrm{C} 1 \mathrm{D}$ & $1.252(6)$ \\
\hline $\mathrm{C} 13 \mathrm{~A}-\mathrm{C} 14 \mathrm{~A}$ & $1.500(7)$ & $\mathrm{N} 1 \mathrm{D}-\mathrm{C} 5 \mathrm{D}$ & $1.380(6)$ \\
\hline $\mathrm{C} 13 \mathrm{~A}-\mathrm{H} 13 \mathrm{~A}$ & 0.9700 & $\mathrm{~N} 1 \mathrm{D}-\mathrm{C} 1 \mathrm{D}$ & $1.399(5)$ \\
\hline $\mathrm{C} 13 \mathrm{~A}-\mathrm{H} 13 \mathrm{~B}$ & 0.9700 & N1D-C9D & $1.480(6)$ \\
\hline $\mathrm{C} 14 \mathrm{~A}-\mathrm{H} 14 \mathrm{~A}$ & 0.9700 & $\mathrm{~N} 2 \mathrm{D}-\mathrm{C} 15 \mathrm{D}$ & $1.492(6)$ \\
\hline $\mathrm{C} 14 \mathrm{~A}-\mathrm{H} 14 \mathrm{~B}$ & 0.9700 & $\mathrm{~N} 2 \mathrm{D}-\mathrm{C} 14 \mathrm{D}$ & $1.509(6)$ \\
\hline $\mathrm{C} 15 \mathrm{~A}-\mathrm{H} 15 \mathrm{~A}$ & 0.9700 & $\mathrm{~N} 2 \mathrm{D}-\mathrm{C} 10 \mathrm{D}$ & $1.515(6)$ \\
\hline $\mathrm{C} 15 \mathrm{~A}-\mathrm{H} 15 \mathrm{~B}$ & 0.9700 & $\mathrm{~N} 2 \mathrm{D}-\mathrm{H} 2 \mathrm{GN}$ & $1.05(5)$ \\
\hline $\mathrm{O} 1 \mathrm{~B}-\mathrm{C} 1 \mathrm{~B}$ & $1.253(6)$ & $\mathrm{C} 1 \mathrm{D}-\mathrm{C} 2 \mathrm{D}$ & $1.413(7)$ \\
\hline $\mathrm{N} 1 \mathrm{~B}-\mathrm{C} 5 \mathrm{~B}$ & $1.379(6)$ & $\mathrm{C} 2 \mathrm{D}-\mathrm{C} 3 \mathrm{D}$ & $1.365(8)$ \\
\hline
\end{tabular}




\begin{tabular}{|c|c|c|c|}
\hline $\mathrm{N} 1 \mathrm{~B}-\mathrm{C} 1 \mathrm{~B}$ & $1.397(6)$ & $\mathrm{C} 2 \mathrm{D}-\mathrm{H} 2 \mathrm{G}$ & 0.9300 \\
\hline $\mathrm{N} 1 \mathrm{~B}-\mathrm{C} 9 \mathrm{~B}$ & $1.490(6)$ & $\mathrm{C} 3 \mathrm{D}-\mathrm{C} 4 \mathrm{D}$ & $1.384(7)$ \\
\hline $\mathrm{N} 2 \mathrm{~B}-\mathrm{C} 15 \mathrm{~B}$ & $1.479(6)$ & $\mathrm{C} 3 \mathrm{D}-\mathrm{H} 3 \mathrm{G}$ & 0.9300 \\
\hline $\mathrm{N} 2 \mathrm{~B}-\mathrm{C} 10 \mathrm{~B}$ & $1.519(6)$ & $C 4 D-C 5 D$ & $1.353(7)$ \\
\hline $\mathrm{N} 2 \mathrm{~B}-\mathrm{C} 14 \mathrm{~B}$ & $1.524(6)$ & $\mathrm{C} 4 \mathrm{D}-\mathrm{H} 4 \mathrm{G}$ & 0.9300 \\
\hline $\mathrm{N} 2 \mathrm{~B}-\mathrm{H} 2 \mathrm{CN}$ & $0.77(6)$ & $\mathrm{C} 5 \mathrm{D}-\mathrm{C} 6 \mathrm{D}$ & $1.519(6)$ \\
\hline $\mathrm{C} 1 \mathrm{~B}-\mathrm{C} 2 \mathrm{~B}$ & $1.412(8)$ & $\mathrm{C} 6 \mathrm{D}-\mathrm{C} 7 \mathrm{D}$ & $1.496(7)$ \\
\hline $\mathrm{C} 2 \mathrm{~B}-\mathrm{C} 3 \mathrm{~B}$ & $1.343(9)$ & $\mathrm{C} 6 \mathrm{D}-\mathrm{C} 15 \mathrm{D}$ & $1.529(7)$ \\
\hline $\mathrm{C} 2 \mathrm{~B}-\mathrm{H} 2 \mathrm{C}$ & 0.9300 & $\mathrm{C} 6 \mathrm{D}-\mathrm{H} 6 \mathrm{G}$ & 0.9800 \\
\hline $\mathrm{C} 3 \mathrm{~B}-\mathrm{C} 4 \mathrm{~B}$ & $1.414(9)$ & $\mathrm{C} 7 \mathrm{D}-\mathrm{C} 8 \mathrm{D}$ & $1.526(8)$ \\
\hline $\mathrm{C} 3 \mathrm{~B}-\mathrm{H} 3 \mathrm{C}$ & 0.9300 & $\mathrm{C} 7 \mathrm{D}-\mathrm{H} 7 \mathrm{G}$ & 0.9700 \\
\hline $\mathrm{C} 4 \mathrm{~B}-\mathrm{C} 5 \mathrm{~B}$ & $1.356(8)$ & $\mathrm{C} 7 \mathrm{D}-\mathrm{H} 7 \mathrm{H}$ & 0.9700 \\
\hline $\mathrm{C} 4 \mathrm{~B}-\mathrm{H} 4 \mathrm{C}$ & 0.9300 & $\mathrm{C} 8 \mathrm{D}-\mathrm{C} 10 \mathrm{D}$ & $1.521(7)$ \\
\hline $\mathrm{C} 5 \mathrm{~B}-\mathrm{C} 6 \mathrm{~B}$ & $1.502(7)$ & $\mathrm{C} 8 \mathrm{D}-\mathrm{C} 9 \mathrm{D}$ & $1.539(6)$ \\
\hline $\mathrm{C} 6 \mathrm{~B}-\mathrm{C} 7 \mathrm{~B}$ & $1.522(9)$ & $\mathrm{C} 8 \mathrm{D}-\mathrm{H} 8 \mathrm{G}$ & 0.9800 \\
\hline $\mathrm{C} 6 \mathrm{~B}-\mathrm{C} 15 \mathrm{~B}$ & $1.541(8)$ & $\mathrm{C} 9 \mathrm{D}-\mathrm{H} 9 \mathrm{G}$ & 0.9700 \\
\hline $\mathrm{C} 6 \mathrm{~B}-\mathrm{H} 6 \mathrm{C}$ & 0.9800 & $\mathrm{C} 9 \mathrm{D}-\mathrm{H} 9 \mathrm{H}$ & 0.9700 \\
\hline $\mathrm{C} 7 \mathrm{~B}-\mathrm{C} 8 \mathrm{~B}$ & $1.516(8)$ & $\mathrm{C} 10 \mathrm{D}-\mathrm{C} 11 \mathrm{D}$ & $1.538(6)$ \\
\hline $\mathrm{C} 7 \mathrm{~B}-\mathrm{H} 7 \mathrm{C}$ & 0.9700 & $\mathrm{C} 10 \mathrm{D}-\mathrm{H} 10 \mathrm{G}$ & 0.9800 \\
\hline $\mathrm{C} 7 \mathrm{~B}-\mathrm{H} 7 \mathrm{D}$ & 0.9700 & $\mathrm{C} 11 \mathrm{D}-\mathrm{C} 12 \mathrm{D}$ & $1.524(9)$ \\
\hline $\mathrm{C} 8 \mathrm{~B}-\mathrm{C} 10 \mathrm{~B}$ & $1.528(8)$ & $\mathrm{C} 11 \mathrm{D}-\mathrm{H} 11 \mathrm{G}$ & 0.9700 \\
\hline $\mathrm{C} 8 \mathrm{~B}-\mathrm{C} 9 \mathrm{~B}$ & $1.529(6)$ & $\mathrm{C} 11 \mathrm{D}-\mathrm{H} 11 \mathrm{H}$ & 0.9700 \\
\hline $\mathrm{C} 8 \mathrm{~B}-\mathrm{H} 8 \mathrm{C}$ & 0.9800 & $\mathrm{C} 12 \mathrm{D}-\mathrm{C} 13 \mathrm{D}$ & $1.514(9)$ \\
\hline $\mathrm{C} 9 \mathrm{~B}-\mathrm{H} 9 \mathrm{C}$ & 0.9700 & $\mathrm{C} 12 \mathrm{D}-\mathrm{H} 12 \mathrm{G}$ & 0.9700 \\
\hline C9B-H9D & 0.9700 & $\mathrm{C} 12 \mathrm{D}-\mathrm{H} 12 \mathrm{H}$ & 0.9700 \\
\hline $\mathrm{C} 10 \mathrm{~B}-\mathrm{C} 11 \mathrm{~B}$ & $1.530(6)$ & $\mathrm{C} 13 \mathrm{D}-\mathrm{C} 14 \mathrm{D}$ & $1.533(7)$ \\
\hline $\mathrm{C} 10 \mathrm{~B}-\mathrm{H} 10 \mathrm{C}$ & 0.9800 & $\mathrm{C} 13 \mathrm{D}-\mathrm{H} 13 \mathrm{G}$ & 0.9700 \\
\hline $\mathrm{C} 11 \mathrm{~B}-\mathrm{C} 12 \mathrm{~B}$ & $1.527(9)$ & $\mathrm{C} 13 \mathrm{D}-\mathrm{H} 13 \mathrm{H}$ & 0.9700 \\
\hline $\mathrm{C} 11 \mathrm{~B}-\mathrm{H} 11 \mathrm{C}$ & 0.9700 & $\mathrm{C} 14 \mathrm{D}-\mathrm{H} 14 \mathrm{G}$ & 0.9700 \\
\hline C11B-H11D & 0.9700 & $\mathrm{C} 14 \mathrm{D}-\mathrm{H} 14 \mathrm{H}$ & 0.9700 \\
\hline $\mathrm{C} 12 \mathrm{~B}-\mathrm{C} 13 \mathrm{~B}$ & $1.490(9)$ & $\mathrm{C} 15 \mathrm{D}-\mathrm{H} 15 \mathrm{G}$ & 0.9700 \\
\hline $\mathrm{C} 12 \mathrm{~B}-\mathrm{H} 12 \mathrm{C}$ & 0.9700 & $\mathrm{C} 15 \mathrm{D}-\mathrm{H} 15 \mathrm{H}$ & 0.9700 \\
\hline $\mathrm{C} 12 \mathrm{~B}-\mathrm{H} 12 \mathrm{D}$ & 0.9700 & $\mathrm{Cl1}-\mathrm{O} 13$ & $1.381(6)$ \\
\hline $\mathrm{C} 13 \mathrm{~B}-\mathrm{C} 14 \mathrm{~B}$ & $1.510(7)$ & $\mathrm{Cl1}-\mathrm{O} 14$ & $1.385(7)$ \\
\hline $\mathrm{C} 13 \mathrm{~B}-\mathrm{H} 13 \mathrm{C}$ & 0.9700 & $\mathrm{Cl1}-\mathrm{O} 11$ & $1.387(7)$ \\
\hline $\mathrm{C} 13 \mathrm{~B}-\mathrm{H} 13 \mathrm{D}$ & 0.9700 & $\mathrm{Cl1}-\mathrm{O} 12$ & $1.393(8)$ \\
\hline $\mathrm{C} 14 \mathrm{~B}-\mathrm{H} 14 \mathrm{C}$ & 0.9700 & $\mathrm{Cl} 2-\mathrm{O} 23$ & $1.285(8)$ \\
\hline $\mathrm{C} 14 \mathrm{~B}-\mathrm{H} 14 \mathrm{D}$ & 0.9700 & $\mathrm{Cl} 2-\mathrm{O} 21$ & $1.290(13)$ \\
\hline $\mathrm{C} 15 \mathrm{~B}-\mathrm{H} 15 \mathrm{C}$ & 0.9700 & $\mathrm{C} 12-\mathrm{O} 22$ & $1.302(8)$ \\
\hline $\mathrm{C} 15 \mathrm{~B}-\mathrm{H} 15 \mathrm{D}$ & 0.9700 & $\mathrm{Cl} 2-\mathrm{O} 24$ & $1.392(6)$ \\
\hline $\mathrm{O} 1 \mathrm{C}-\mathrm{C} 1 \mathrm{C}$ & $1.243(6)$ & $\mathrm{Cl} 3-\mathrm{O} 31$ & $1.377(7)$ \\
\hline $\mathrm{N} 1 \mathrm{C}-\mathrm{C} 5 \mathrm{C}$ & $1.394(6)$ & $\mathrm{Cl} 3-\mathrm{O} 32$ & $1.385(9)$ \\
\hline $\mathrm{N} 1 \mathrm{C}-\mathrm{C} 1 \mathrm{C}$ & $1.395(6)$ & $\mathrm{Cl} 3-\mathrm{O} 33$ & $1.389(5)$ \\
\hline $\mathrm{N} 1 \mathrm{C}-\mathrm{C} 9 \mathrm{C}$ & $1.486(6)$ & $\mathrm{Cl} 3-\mathrm{O} 34$ & $1.390(8)$ \\
\hline $\mathrm{N} 2 \mathrm{C}-\mathrm{C} 15 \mathrm{C}$ & $1.502(6)$ & $\mathrm{Cl} 4-\mathrm{O} 42$ & $1.362(8)$ \\
\hline $\mathrm{N} 2 \mathrm{C}-\mathrm{C} 14 \mathrm{C}$ & $1.510(7)$ & $\mathrm{Cl} 4-\mathrm{O} 43$ & $1.391(5)$ \\
\hline $\mathrm{N} 2 \mathrm{C}-\mathrm{C} 10 \mathrm{C}$ & $1.527(6)$ & $\mathrm{Cl} 4-\mathrm{O} 41$ & $1.400(10)$ \\
\hline $\mathrm{N} 2 \mathrm{C}-\mathrm{H} 2 \mathrm{EN}$ & $0.91(9)$ & $\mathrm{Cl} 4-\mathrm{O} 44$ & $1.404(5)$ \\
\hline
\end{tabular}




\begin{tabular}{|c|c|c|c|}
\hline $\mathrm{C} 5 \mathrm{~A}-\mathrm{N} 1 \mathrm{~A}-\mathrm{C} 1 \mathrm{~A}$ & $121.9(4)$ & $\mathrm{C} 3 \mathrm{C}-\mathrm{C} 2 \mathrm{C}-\mathrm{C} 1 \mathrm{C}$ & $121.4(4)$ \\
\hline $\mathrm{C} 5 \mathrm{~A}-\mathrm{N} 1 \mathrm{~A}-\mathrm{C} 9 \mathrm{~A}$ & $122.9(4)$ & $\mathrm{C} 3 \mathrm{C}-\mathrm{C} 2 \mathrm{C}-\mathrm{H} 2 \mathrm{E}$ & 119.3 \\
\hline $\mathrm{C} 1 \mathrm{~A}-\mathrm{N} 1 \mathrm{~A}-\mathrm{C} 9 \mathrm{~A}$ & $115.2(4)$ & $\mathrm{C} 1 \mathrm{C}-\mathrm{C} 2 \mathrm{C}-\mathrm{H} 2 \mathrm{E}$ & 119.3 \\
\hline $\mathrm{C} 15 \mathrm{~A}-\mathrm{N} 2 \mathrm{~A}-\mathrm{C} 14 \mathrm{~A}$ & $112.0(4)$ & $\mathrm{C} 2 \mathrm{C}-\mathrm{C} 3 \mathrm{C}-\mathrm{C} 4 \mathrm{C}$ & $120.5(5)$ \\
\hline $\mathrm{C} 15 \mathrm{~A}-\mathrm{N} 2 \mathrm{~A}-\mathrm{C} 10 \mathrm{~A}$ & $112.9(4)$ & $\mathrm{C} 2 \mathrm{C}-\mathrm{C} 3 \mathrm{C}-\mathrm{H} 3 \mathrm{E}$ & 119.8 \\
\hline $\mathrm{C} 14 \mathrm{~A}-\mathrm{N} 2 \mathrm{~A}-\mathrm{C} 10 \mathrm{~A}$ & $112.1(4)$ & $\mathrm{C} 4 \mathrm{C}-\mathrm{C} 3 \mathrm{C}-\mathrm{H} 3 \mathrm{E}$ & 119.8 \\
\hline $\mathrm{C} 15 \mathrm{~A}-\mathrm{N} 2 \mathrm{~A}-\mathrm{H} 2 \mathrm{AN}$ & $104(3)$ & $\mathrm{C} 5 \mathrm{C}-\mathrm{C} 4 \mathrm{C}-\mathrm{C} 3 \mathrm{C}$ & $120.4(5)$ \\
\hline $\mathrm{C} 14 \mathrm{~A}-\mathrm{N} 2 \mathrm{~A}-\mathrm{H} 2 \mathrm{AN}$ & $101(3)$ & $\mathrm{C} 5 \mathrm{C}-\mathrm{C} 4 \mathrm{C}-\mathrm{H} 4 \mathrm{E}$ & 119.8 \\
\hline $\mathrm{C} 10 \mathrm{~A}-\mathrm{N} 2 \mathrm{~A}-\mathrm{H} 2 \mathrm{AN}$ & $114(3)$ & $\mathrm{C} 3 \mathrm{C}-\mathrm{C} 4 \mathrm{C}-\mathrm{H} 4 \mathrm{E}$ & 119.8 \\
\hline $\mathrm{O} 1 \mathrm{~A}-\mathrm{C} 1 \mathrm{~A}-\mathrm{N} 1 \mathrm{~A}$ & $118.1(5)$ & $\mathrm{C} 4 \mathrm{C}-\mathrm{C} 5 \mathrm{C}-\mathrm{N} 1 \mathrm{C}$ & $119.5(4)$ \\
\hline $\mathrm{O} 1 \mathrm{~A}-\mathrm{C} 1 \mathrm{~A}-\mathrm{C} 2 \mathrm{~A}$ & $125.0(5)$ & $\mathrm{C} 4 \mathrm{C}-\mathrm{C} 5 \mathrm{C}-\mathrm{C} 6 \mathrm{C}$ & $122.9(4)$ \\
\hline $\mathrm{N} 1 \mathrm{~A}-\mathrm{C} 1 \mathrm{~A}-\mathrm{C} 2 \mathrm{~A}$ & $116.9(4)$ & $\mathrm{N} 1 \mathrm{C}-\mathrm{C} 5 \mathrm{C}-\mathrm{C} 6 \mathrm{C}$ & $117.5(4)$ \\
\hline $\mathrm{C} 3 \mathrm{~A}-\mathrm{C} 2 \mathrm{~A}-\mathrm{C} 1 \mathrm{~A}$ & $121.1(5)$ & $\mathrm{C} 5 \mathrm{C}-\mathrm{C} 6 \mathrm{C}-\mathrm{C} 15 \mathrm{C}$ & $111.5(4)$ \\
\hline $\mathrm{C} 3 \mathrm{~A}-\mathrm{C} 2 \mathrm{~A}-\mathrm{H} 2 \mathrm{~A}$ & 119.4 & $\mathrm{C} 5 \mathrm{C}-\mathrm{C} 6 \mathrm{C}-\mathrm{C} 7 \mathrm{C}$ & $111.4(4)$ \\
\hline $\mathrm{C} 1 \mathrm{~A}-\mathrm{C} 2 \mathrm{~A}-\mathrm{H} 2 \mathrm{~A}$ & 119.4 & $\mathrm{C} 15 \mathrm{C}-\mathrm{C} 6 \mathrm{C}-\mathrm{C} 7 \mathrm{C}$ & $110.7(4)$ \\
\hline $\mathrm{C} 2 \mathrm{~A}-\mathrm{C} 3 \mathrm{~A}-\mathrm{C} 4 \mathrm{~A}$ & $120.2(5)$ & $\mathrm{C} 5 \mathrm{C}-\mathrm{C} 6 \mathrm{C}-\mathrm{H} 6 \mathrm{E}$ & 107.7 \\
\hline $\mathrm{C} 2 \mathrm{~A}-\mathrm{C} 3 \mathrm{~A}-\mathrm{H} 3 \mathrm{~A}$ & 119.9 & $\mathrm{C} 15 \mathrm{C}-\mathrm{C} 6 \mathrm{C}-\mathrm{H} 6 \mathrm{E}$ & 107.7 \\
\hline $\mathrm{C} 4 \mathrm{~A}-\mathrm{C} 3 \mathrm{~A}-\mathrm{H} 3 \mathrm{~A}$ & 119.9 & $\mathrm{C} 7 \mathrm{C}-\mathrm{C} 6 \mathrm{C}-\mathrm{H} 6 \mathrm{E}$ & 107.7 \\
\hline $\mathrm{C} 5 \mathrm{~A}-\mathrm{C} 4 \mathrm{~A}-\mathrm{C} 3 \mathrm{~A}$ & $120.2(5)$ & $\mathrm{C} 8 \mathrm{C}-\mathrm{C} 7 \mathrm{C}-\mathrm{C} 6 \mathrm{C}$ & $106.1(4)$ \\
\hline $\mathrm{C} 5 \mathrm{~A}-\mathrm{C} 4 \mathrm{~A}-\mathrm{H} 4 \mathrm{~A}$ & 119.9 & $\mathrm{C} 8 \mathrm{C}-\mathrm{C} 7 \mathrm{C}-\mathrm{H} 7 \mathrm{E}$ & 110.5 \\
\hline $\mathrm{C} 3 \mathrm{~A}-\mathrm{C} 4 \mathrm{~A}-\mathrm{H} 4 \mathrm{~A}$ & 119.9 & $\mathrm{C} 6 \mathrm{C}-\mathrm{C} 7 \mathrm{C}-\mathrm{H} 7 \mathrm{E}$ & 110.5 \\
\hline $\mathrm{C} 4 \mathrm{~A}-\mathrm{C} 5 \mathrm{~A}-\mathrm{N} 1 \mathrm{~A}$ & $119.6(4)$ & $\mathrm{C} 8 \mathrm{C}-\mathrm{C} 7 \mathrm{C}-\mathrm{H} 7 \mathrm{~F}$ & 110.5 \\
\hline $\mathrm{C} 4 \mathrm{~A}-\mathrm{C} 5 \mathrm{~A}-\mathrm{C} 6 \mathrm{~A}$ & $121.7(4)$ & $\mathrm{C} 6 \mathrm{C}-\mathrm{C} 7 \mathrm{C}-\mathrm{H} 7 \mathrm{~F}$ & 110.5 \\
\hline $\mathrm{N} 1 \mathrm{~A}-\mathrm{C} 5 \mathrm{~A}-\mathrm{C} 6 \mathrm{~A}$ & $118.6(4)$ & $\mathrm{H} 7 \mathrm{E}-\mathrm{C} 7 \mathrm{C}-\mathrm{H} 7 \mathrm{~F}$ & 108.7 \\
\hline $\mathrm{C} 5 \mathrm{~A}-\mathrm{C} 6 \mathrm{~A}-\mathrm{C} 7 \mathrm{~A}$ & $111.0(4)$ & $\mathrm{C} 7 \mathrm{C}-\mathrm{C} 8 \mathrm{C}-\mathrm{C} 9 \mathrm{C}$ & $109.3(4)$ \\
\hline $\mathrm{C} 5 \mathrm{~A}-\mathrm{C} 6 \mathrm{~A}-\mathrm{C} 15 \mathrm{~A}$ & $111.0(4)$ & $\mathrm{C} 7 \mathrm{C}-\mathrm{C} 8 \mathrm{C}-\mathrm{C} 10 \mathrm{C}$ & $112.9(4)$ \\
\hline $\mathrm{C} 7 \mathrm{~A}-\mathrm{C} 6 \mathrm{~A}-\mathrm{C} 15 \mathrm{~A}$ & $110.3(4)$ & $\mathrm{C} 9 \mathrm{C}-\mathrm{C} 8 \mathrm{C}-\mathrm{C} 10 \mathrm{C}$ & $113.6(4)$ \\
\hline $\mathrm{C} 5 \mathrm{~A}-\mathrm{C} 6 \mathrm{~A}-\mathrm{H} 6 \mathrm{~A}$ & 108.2 & $\mathrm{C} 7 \mathrm{C}-\mathrm{C} 8 \mathrm{C}-\mathrm{H} 8 \mathrm{E}$ & 106.8 \\
\hline $\mathrm{C} 7 \mathrm{~A}-\mathrm{C} 6 \mathrm{~A}-\mathrm{H} 6 \mathrm{~A}$ & 108.2 & $\mathrm{C} 9 \mathrm{C}-\mathrm{C} 8 \mathrm{C}-\mathrm{H} 8 \mathrm{E}$ & 106.8 \\
\hline $\mathrm{C} 15 \mathrm{~A}-\mathrm{C} 6 \mathrm{~A}-\mathrm{H} 6 \mathrm{~A}$ & 108.2 & $\mathrm{C} 10 \mathrm{C}-\mathrm{C} 8 \mathrm{C}-\mathrm{H} 8 \mathrm{E}$ & 106.8 \\
\hline $\mathrm{C} 6 \mathrm{~A}-\mathrm{C} 7 \mathrm{~A}-\mathrm{C} 8 \mathrm{~A}$ & $106.3(4)$ & $\mathrm{N} 1 \mathrm{C}-\mathrm{C} 9 \mathrm{C}-\mathrm{C} 8 \mathrm{C}$ & $115.3(4)$ \\
\hline $\mathrm{C} 6 \mathrm{~A}-\mathrm{C} 7 \mathrm{~A}-\mathrm{H} 7 \mathrm{~A}$ & 110.5 & $\mathrm{~N} 1 \mathrm{C}-\mathrm{C} 9 \mathrm{C}-\mathrm{H} 9 \mathrm{E}$ & 108.5 \\
\hline $\mathrm{C} 8 \mathrm{~A}-\mathrm{C} 7 \mathrm{~A}-\mathrm{H} 7 \mathrm{~A}$ & 110.5 & $\mathrm{C} 8 \mathrm{C}-\mathrm{C} 9 \mathrm{C}-\mathrm{H} 9 \mathrm{E}$ & 108.5 \\
\hline $\mathrm{C} 6 \mathrm{~A}-\mathrm{C} 7 \mathrm{~A}-\mathrm{H} 7 \mathrm{~B}$ & 110.5 & $\mathrm{~N} 1 \mathrm{C}-\mathrm{C} 9 \mathrm{C}-\mathrm{H} 9 \mathrm{~F}$ & 108.5 \\
\hline $\mathrm{C} 8 \mathrm{~A}-\mathrm{C} 7 \mathrm{~A}-\mathrm{H} 7 \mathrm{~B}$ & 110.5 & $\mathrm{C} 8 \mathrm{C}-\mathrm{C} 9 \mathrm{C}-\mathrm{H} 9 \mathrm{~F}$ & 108.5 \\
\hline $\mathrm{H} 7 \mathrm{~A}-\mathrm{C} 7 \mathrm{~A}-\mathrm{H} 7 \mathrm{~B}$ & 108.7 & $\mathrm{H} 9 \mathrm{E}-\mathrm{C} 9 \mathrm{C}-\mathrm{H} 9 \mathrm{~F}$ & 107.5 \\
\hline $\mathrm{C} 7 \mathrm{~A}-\mathrm{C} 8 \mathrm{~A}-\mathrm{C} 9 \mathrm{~A}$ & $109.1(4)$ & $\mathrm{N} 2 \mathrm{C}-\mathrm{C} 10 \mathrm{C}-\mathrm{C} 11 \mathrm{C}$ & $110.6(4)$ \\
\hline $\mathrm{C} 7 \mathrm{~A}-\mathrm{C} 8 \mathrm{~A}-\mathrm{C} 10 \mathrm{~A}$ & $111.9(4)$ & $\mathrm{N} 2 \mathrm{C}-\mathrm{C} 10 \mathrm{C}-\mathrm{C} 8 \mathrm{C}$ & $108.9(4)$ \\
\hline $\mathrm{C} 9 \mathrm{~A}-\mathrm{C} 8 \mathrm{~A}-\mathrm{C} 10 \mathrm{~A}$ & $113.7(4)$ & $\mathrm{C} 11 \mathrm{C}-\mathrm{C} 10 \mathrm{C}-\mathrm{C} 8 \mathrm{C}$ & $114.1(5)$ \\
\hline $\mathrm{C} 7 \mathrm{~A}-\mathrm{C} 8 \mathrm{~A}-\mathrm{H} 8 \mathrm{~A}$ & 107.3 & $\mathrm{~N} 2 \mathrm{C}-\mathrm{C} 10 \mathrm{C}-\mathrm{H} 10 \mathrm{E}$ & 107.7 \\
\hline $\mathrm{C} 9 \mathrm{~A}-\mathrm{C} 8 \mathrm{~A}-\mathrm{H} 8 \mathrm{~A}$ & 107.3 & $\mathrm{C} 11 \mathrm{C}-\mathrm{C} 10 \mathrm{C}-\mathrm{H} 10 \mathrm{E}$ & 107.7 \\
\hline $\mathrm{C} 10 \mathrm{~A}-\mathrm{C} 8 \mathrm{~A}-\mathrm{H} 8 \mathrm{~A}$ & 107.3 & $\mathrm{C} 8 \mathrm{C}-\mathrm{C} 10 \mathrm{C}-\mathrm{H} 10 \mathrm{E}$ & 107.7 \\
\hline $\mathrm{N} 1 \mathrm{~A}-\mathrm{C} 9 \mathrm{~A}-\mathrm{C} 8 \mathrm{~A}$ & $115.0(4)$ & $\mathrm{C} 12 \mathrm{C}-\mathrm{C} 11 \mathrm{C}-\mathrm{C} 10 \mathrm{C}$ & $113.1(5)$ \\
\hline $\mathrm{N} 1 \mathrm{~A}-\mathrm{C} 9 \mathrm{~A}-\mathrm{H} 9 \mathrm{~A}$ & 108.5 & $\mathrm{C} 12 \mathrm{C}-\mathrm{C} 11 \mathrm{C}-\mathrm{H} 11 \mathrm{E}$ & 109.0 \\
\hline $\mathrm{C} 8 \mathrm{~A}-\mathrm{C} 9 \mathrm{~A}-\mathrm{H} 9 \mathrm{~A}$ & 108.5 & $\mathrm{C} 10 \mathrm{C}-\mathrm{C} 11 \mathrm{C}-\mathrm{H} 11 \mathrm{E}$ & 109.0 \\
\hline $\mathrm{N} 1 \mathrm{~A}-\mathrm{C} 9 \mathrm{~A}-\mathrm{H} 9 \mathrm{~B}$ & 108.5 & $\mathrm{C} 12 \mathrm{C}-\mathrm{C} 11 \mathrm{C}-\mathrm{H} 11 \mathrm{~F}$ & 109.0 \\
\hline $\mathrm{C} 8 \mathrm{~A}-\mathrm{C} 9 \mathrm{~A}-\mathrm{H} 9 \mathrm{~B}$ & 108.5 & $\mathrm{C} 10 \mathrm{C}-\mathrm{C} 11 \mathrm{C}-\mathrm{H} 11 \mathrm{~F}$ & 109.0 \\
\hline
\end{tabular}




$\begin{array}{ll}\text { H9A-C9A-H9B } & 107.5 \\ \text { N2A-C10A-C11A } & 111.1(4) \\ \text { N2A-C10A-C8A } & 109.6(4) \\ \text { C11A-C10A-C8A } & 113.4(5) \\ \text { N2A-C10A-H10A } & 107.5 \\ \text { C11A-C10A-H10A } & 107.5 \\ \text { C8A-C10A-H10A } & 107.5 \\ \text { C10A-C11A-C12A } & 111.3(5) \\ \text { C10A-C11A-H11A } & 109.4 \\ \text { C12A-C11A-H11A } & 109.4 \\ \text { C10A-C11A-H11B } & 109.4 \\ \text { C12A-C11A-H11B } & 109.4 \\ \text { H11A-C11A-H11B } & 108.0 \\ \text { C13A-C12A-C11A } & 111.8(5) \\ \text { C13A-C12A-H12A } & 109.3 \\ \text { C11A-C12A-H12A } & 109.3 \\ \text { C13A-C12A-H12B } & 109.3 \\ \text { C11A-C12A-H12B } & 109.3 \\ \text { H12A-C12A-H12B } & 107.9 \\ \text { C14A-C13A-C12A } & 110.5(5) \\ \text { C14A-C13A-H13A } & 109.6 \\ \text { C12A-C13A-H13A } & 109.6 \\ \text { C14A-C13A-H13B } & 109.6 \\ \text { C12A-C13A-H13B } & 109.6 \\ \text { H13A-C13A-H13B } & 108.1 \\ \text { C13A-C14A-N2A } & 112.9(5) \\ \text { C13A-C14A-H14A } & 109.0 \\ \text { N2A-C14A-H14A } & 109.0 \\ \text { C13A-C14A-H14B } & 109.0 \\ \text { N2A-C14A-H14B } & 109.0 \\ \text { H14A-C14A-H14B } & 107.8 \\ \text { N2A-C15A-C6A } & 111.8(4) \\ \text { N2A-C15A-H15A } & 109.3 \\ \text { C6A-C15A-H15A } & 109.3 \\ \text { N2A-C15A-H15B } & 109.3 \\ \text { C6A-C15A-H15B } & 109.3 \\ \text { H15A-C15A-H15B } & 107.9 \\ \text { C5B-N1B-C1B } & 122.2(4) \\ \text { C5B-N1B-C9B } & 123.3(4) \\ \text { C1B-N1B-C9B } & 114.6(4) \\ \text { C15B-N2B-C10B } & 114.9(4) \\ \text { C15B-N2B-C14B } & 111.9(4) \\ \text { C10B-N2B-C14B } & 111.6(4) \\ \text { C15B-N2B-H2CN } & 111(4) \\ \text { C10B-N2B-H2CN } & 107(4) \\ \text { C14B-N2B-H2CN } & 100(5) \\ \text { O1B-C1B-N1B } & 118.3(5) \\ \text { O1B-C1B-C2B } & 125.4(5) \\ & \end{array}$

$\begin{array}{ll}\mathrm{H} 11 \mathrm{E}-\mathrm{C} 11 \mathrm{C}-\mathrm{H} 11 \mathrm{~F} & 107.8 \\ \mathrm{C} 13 \mathrm{C}-\mathrm{C} 12 \mathrm{C}-\mathrm{C} 11 \mathrm{C} & 111.4(5) \\ \mathrm{C} 13 \mathrm{C}-\mathrm{C} 12 \mathrm{C}-\mathrm{H} 12 \mathrm{E} & 109.3 \\ \mathrm{C} 11 \mathrm{C}-\mathrm{C} 12 \mathrm{C}-\mathrm{H} 12 \mathrm{E} & 109.3 \\ \mathrm{C} 13 \mathrm{C}-\mathrm{C} 12 \mathrm{C}-\mathrm{H} 12 \mathrm{~F} & 109.3 \\ \mathrm{C} 11 \mathrm{C}-\mathrm{C} 12 \mathrm{C}-\mathrm{H} 12 \mathrm{~F} & 109.3 \\ \mathrm{H} 12 \mathrm{E}-\mathrm{C} 12 \mathrm{C}-\mathrm{H} 12 \mathrm{~F} & 108.0 \\ \mathrm{C} 12 \mathrm{C}-\mathrm{C} 13 \mathrm{C}-\mathrm{C} 14 \mathrm{C} & 109.7(5) \\ \mathrm{C} 12 \mathrm{C}-\mathrm{C} 13 \mathrm{C}-\mathrm{H} 13 \mathrm{E} & 109.7 \\ \mathrm{C} 14 \mathrm{C}-\mathrm{C} 13 \mathrm{C}-\mathrm{H} 13 \mathrm{E} & 109.7 \\ \mathrm{C} 12 \mathrm{C}-\mathrm{C} 13 \mathrm{C}-\mathrm{H} 13 \mathrm{~F} & 109.7 \\ \mathrm{C} 14 \mathrm{C}-\mathrm{C} 13 \mathrm{C}-\mathrm{H} 13 \mathrm{~F} & 109.7 \\ \mathrm{H} 13 \mathrm{E}-\mathrm{C} 13 \mathrm{C}-\mathrm{H} 13 \mathrm{~F} & 108.2 \\ \mathrm{~N} 2 \mathrm{C}-\mathrm{C} 14 \mathrm{C}-\mathrm{C} 13 \mathrm{C} & 113.3(5) \\ \mathrm{N} 2 \mathrm{C}-\mathrm{C} 14 \mathrm{C}-\mathrm{H} 14 \mathrm{E} & 108.9\end{array}$

$\mathrm{N} 2 \mathrm{C}-\mathrm{C} 14 \mathrm{C}-\mathrm{H} 14 \mathrm{E} \quad 108.9$

$\mathrm{C} 13 \mathrm{C}-\mathrm{C} 14 \mathrm{C}-\mathrm{H} 14 \mathrm{E} \quad 108.9$

$\mathrm{N} 2 \mathrm{C}-\mathrm{C} 14 \mathrm{C}-\mathrm{H} 14 \mathrm{~F} \quad 108.9$

$\mathrm{C} 13 \mathrm{C}-\mathrm{C} 14 \mathrm{C}-\mathrm{H} 14 \mathrm{~F} \quad 108.9$

$\mathrm{H} 14 \mathrm{E}-\mathrm{C} 14 \mathrm{C}-\mathrm{H} 14 \mathrm{~F} \quad 107.7$

$\mathrm{N} 2 \mathrm{C}-\mathrm{C} 15 \mathrm{C}-\mathrm{C} 6 \mathrm{C} \quad 112.0(4)$

$\mathrm{N} 2 \mathrm{C}-\mathrm{C} 15 \mathrm{C}-\mathrm{H} 15 \mathrm{E} \quad 109.2$

$\mathrm{C} 6 \mathrm{C}-\mathrm{C} 15 \mathrm{C}-\mathrm{H} 15 \mathrm{E} \quad 109.2$

$\mathrm{N} 2 \mathrm{C}-\mathrm{C} 15 \mathrm{C}-\mathrm{H} 15 \mathrm{~F} \quad 109.2$

$\mathrm{C} 6 \mathrm{C}-\mathrm{C} 15 \mathrm{C}-\mathrm{H} 15 \mathrm{~F} \quad 109.2$

$\mathrm{H} 15 \mathrm{E}-\mathrm{C} 15 \mathrm{C}-\mathrm{H} 15 \mathrm{~F} \quad 107.9$

C5D-N1D-C1D 121.8 (4)

C5D-N1D-C9D 123.8 (4)

C1D-N1D-C9D 114.4 (4)

C15D-N2D-C14D 111.9 (4)

C15D-N2D-C10D 113.7 (4)

C14D-N2D-C10D 113.0 (4)

C15D-N2D-H2GN 111 (3)

C14D-N2D-H2GN 104 (3)

C10D-N2D-H2GN 103 (3)

O1D-C1D-N1D $118.4(4)$

O1D - C1D - C2D $125.3(4)$

N1D-C1D-C2D 116.4 (4)

$\mathrm{C} 3 \mathrm{D}-\mathrm{C} 2 \mathrm{D}-\mathrm{C} 1 \mathrm{D} \quad 120.9(5)$

$\mathrm{C} 3 \mathrm{D}-\mathrm{C} 2 \mathrm{D}-\mathrm{H} 2 \mathrm{G} \quad 119.6$

$\mathrm{C} 1 \mathrm{D}-\mathrm{C} 2 \mathrm{D}-\mathrm{H} 2 \mathrm{G} \quad 119.6$

C2D-C3D-C4D 120.7 (5)

$\mathrm{C} 2 \mathrm{D}-\mathrm{C} 3 \mathrm{D}-\mathrm{H} 3 \mathrm{G} \quad 119.6$

$\mathrm{C} 4 \mathrm{D}-\mathrm{C} 3 \mathrm{D}-\mathrm{H} 3 \mathrm{G} \quad 119.6$

$\mathrm{C} 5 \mathrm{D}-\mathrm{C} 4 \mathrm{D}-\mathrm{C} 3 \mathrm{D} \quad 119.9$ (5)

$\mathrm{C} 5 \mathrm{D}-\mathrm{C} 4 \mathrm{D}-\mathrm{H} 4 \mathrm{G} \quad 120.1$

C3D-C4D-H4G $\quad 120.1$

C4D-C5D-N1D $120.2(4)$

C4D-C5D-C6D 121.8 (4) 


\begin{tabular}{|c|c|c|c|}
\hline $\mathrm{N} 1 \mathrm{~B}-\mathrm{C} 1 \mathrm{~B}-\mathrm{C} 2 \mathrm{~B}$ & $116.3(5)$ & $\mathrm{N} 1 \mathrm{D}-\mathrm{C} 5 \mathrm{D}-\mathrm{C} 6 \mathrm{D}$ & $118.0(4)$ \\
\hline $\mathrm{C} 3 \mathrm{~B}-\mathrm{C} 2 \mathrm{~B}-\mathrm{C} 1 \mathrm{~B}$ & $121.5(5)$ & $\mathrm{C} 7 \mathrm{D}-\mathrm{C} 6 \mathrm{D}-\mathrm{C} 5 \mathrm{D}$ & $111.8(4)$ \\
\hline $\mathrm{C} 3 \mathrm{~B}-\mathrm{C} 2 \mathrm{~B}-\mathrm{H} 2 \mathrm{C}$ & 119.3 & $\mathrm{C} 7 \mathrm{D}-\mathrm{C} 6 \mathrm{D}-\mathrm{C} 15 \mathrm{D}$ & $110.4(4)$ \\
\hline $\mathrm{C} 1 \mathrm{~B}-\mathrm{C} 2 \mathrm{~B}-\mathrm{H} 2 \mathrm{C}$ & 119.3 & $\mathrm{C} 5 \mathrm{D}-\mathrm{C} 6 \mathrm{D}-\mathrm{C} 15 \mathrm{D}$ & $110.0(4)$ \\
\hline $\mathrm{C} 2 \mathrm{~B}-\mathrm{C} 3 \mathrm{~B}-\mathrm{C} 4 \mathrm{~B}$ & $120.9(6)$ & $\mathrm{C} 7 \mathrm{D}-\mathrm{C} 6 \mathrm{D}-\mathrm{H} 6 \mathrm{G}$ & 108.2 \\
\hline $\mathrm{C} 2 \mathrm{~B}-\mathrm{C} 3 \mathrm{~B}-\mathrm{H} 3 \mathrm{C}$ & 119.6 & $\mathrm{C} 5 \mathrm{D}-\mathrm{C} 6 \mathrm{D}-\mathrm{H} 6 \mathrm{G}$ & 108.2 \\
\hline $\mathrm{C} 4 \mathrm{~B}-\mathrm{C} 3 \mathrm{~B}-\mathrm{H} 3 \mathrm{C}$ & 119.6 & $\mathrm{C} 15 \mathrm{D}-\mathrm{C} 6 \mathrm{D}-\mathrm{H} 6 \mathrm{G}$ & 108.2 \\
\hline $\mathrm{C} 5 \mathrm{~B}-\mathrm{C} 4 \mathrm{~B}-\mathrm{C} 3 \mathrm{~B}$ & $118.8(5)$ & $\mathrm{C} 6 \mathrm{D}-\mathrm{C} 7 \mathrm{D}-\mathrm{C} 8 \mathrm{D}$ & $107.7(4)$ \\
\hline $\mathrm{C} 5 \mathrm{~B}-\mathrm{C} 4 \mathrm{~B}-\mathrm{H} 4 \mathrm{C}$ & 120.6 & $\mathrm{C} 6 \mathrm{D}-\mathrm{C} 7 \mathrm{D}-\mathrm{H} 7 \mathrm{G}$ & 110.2 \\
\hline $\mathrm{C} 3 \mathrm{~B}-\mathrm{C} 4 \mathrm{~B}-\mathrm{H} 4 \mathrm{C}$ & 120.6 & $\mathrm{C} 8 \mathrm{D}-\mathrm{C} 7 \mathrm{D}-\mathrm{H} 7 \mathrm{G}$ & 110.2 \\
\hline $\mathrm{C} 4 \mathrm{~B}-\mathrm{C} 5 \mathrm{~B}-\mathrm{N} 1 \mathrm{~B}$ & $120.3(5)$ & $\mathrm{C} 6 \mathrm{D}-\mathrm{C} 7 \mathrm{D}-\mathrm{H} 7 \mathrm{H}$ & 110.2 \\
\hline $\mathrm{C} 4 \mathrm{~B}-\mathrm{C} 5 \mathrm{~B}-\mathrm{C} 6 \mathrm{~B}$ & $120.8(5)$ & $\mathrm{C} 8 \mathrm{D}-\mathrm{C} 7 \mathrm{D}-\mathrm{H} 7 \mathrm{H}$ & 110.2 \\
\hline $\mathrm{N} 1 \mathrm{~B}-\mathrm{C} 5 \mathrm{~B}-\mathrm{C} 6 \mathrm{~B}$ & $118.9(5)$ & $\mathrm{H} 7 \mathrm{G}-\mathrm{C} 7 \mathrm{D}-\mathrm{H} 7 \mathrm{H}$ & 108.5 \\
\hline $\mathrm{C} 5 \mathrm{~B}-\mathrm{C} 6 \mathrm{~B}-\mathrm{C} 7 \mathrm{~B}$ & $111.2(4)$ & $\mathrm{C} 10 \mathrm{D}-\mathrm{C} 8 \mathrm{D}-\mathrm{C} 7 \mathrm{D}$ & $111.0(4)$ \\
\hline $\mathrm{C} 5 \mathrm{~B}-\mathrm{C} 6 \mathrm{~B}-\mathrm{C} 15 \mathrm{~B}$ & $110.9(4)$ & $\mathrm{C} 10 \mathrm{D}-\mathrm{C} 8 \mathrm{D}-\mathrm{C} 9 \mathrm{D}$ & $113.8(4)$ \\
\hline $\mathrm{C} 7 \mathrm{~B}-\mathrm{C} 6 \mathrm{~B}-\mathrm{C} 15 \mathrm{~B}$ & $109.3(5)$ & $\mathrm{C} 7 \mathrm{D}-\mathrm{C} 8 \mathrm{D}-\mathrm{C} 9 \mathrm{D}$ & $109.0(4)$ \\
\hline $\mathrm{C} 5 \mathrm{~B}-\mathrm{C} 6 \mathrm{~B}-\mathrm{H} 6 \mathrm{C}$ & 108.5 & $\mathrm{C} 10 \mathrm{D}-\mathrm{C} 8 \mathrm{D}-\mathrm{H} 8 \mathrm{G}$ & 107.6 \\
\hline $\mathrm{C} 7 \mathrm{~B}-\mathrm{C} 6 \mathrm{~B}-\mathrm{H} 6 \mathrm{C}$ & 108.5 & $\mathrm{C} 7 \mathrm{D}-\mathrm{C} 8 \mathrm{D}-\mathrm{H} 8 \mathrm{G}$ & 107.6 \\
\hline $\mathrm{C} 15 \mathrm{~B}-\mathrm{C} 6 \mathrm{~B}-\mathrm{H} 6 \mathrm{C}$ & 108.5 & $\mathrm{C} 9 \mathrm{D}-\mathrm{C} 8 \mathrm{D}-\mathrm{H} 8 \mathrm{G}$ & 107.6 \\
\hline $\mathrm{C} 8 \mathrm{~B}-\mathrm{C} 7 \mathrm{~B}-\mathrm{C} 6 \mathrm{~B}$ & $107.1(4)$ & $\mathrm{N} 1 \mathrm{D}-\mathrm{C} 9 \mathrm{D}-\mathrm{C} 8 \mathrm{D}$ & $115.0(4)$ \\
\hline $\mathrm{C} 8 \mathrm{~B}-\mathrm{C} 7 \mathrm{~B}-\mathrm{H} 7 \mathrm{C}$ & 110.3 & $\mathrm{~N} 1 \mathrm{D}-\mathrm{C} 9 \mathrm{D}-\mathrm{H} 9 \mathrm{G}$ & 108.5 \\
\hline $\mathrm{C} 6 \mathrm{~B}-\mathrm{C} 7 \mathrm{~B}-\mathrm{H} 7 \mathrm{C}$ & 110.3 & $\mathrm{C} 8 \mathrm{D}-\mathrm{C} 9 \mathrm{D}-\mathrm{H} 9 \mathrm{G}$ & 108.5 \\
\hline $\mathrm{C} 8 \mathrm{~B}-\mathrm{C} 7 \mathrm{~B}-\mathrm{H} 7 \mathrm{D}$ & 110.3 & $\mathrm{~N} 1 \mathrm{D}-\mathrm{C} 9 \mathrm{D}-\mathrm{H} 9 \mathrm{H}$ & 108.5 \\
\hline $\mathrm{C} 6 \mathrm{~B}-\mathrm{C} 7 \mathrm{~B}-\mathrm{H} 7 \mathrm{D}$ & 110.3 & $\mathrm{C} 8 \mathrm{D}-\mathrm{C} 9 \mathrm{D}-\mathrm{H} 9 \mathrm{H}$ & 108.5 \\
\hline $\mathrm{H} 7 \mathrm{C}-\mathrm{C} 7 \mathrm{~B}-\mathrm{H} 7 \mathrm{D}$ & 108.5 & $\mathrm{H} 9 \mathrm{G}-\mathrm{C} 9 \mathrm{D}-\mathrm{H} 9 \mathrm{H}$ & 107.5 \\
\hline $\mathrm{C} 7 \mathrm{~B}-\mathrm{C} 8 \mathrm{~B}-\mathrm{C} 10 \mathrm{~B}$ & $111.5(4)$ & $\mathrm{N} 2 \mathrm{D}-\mathrm{C} 10 \mathrm{D}-\mathrm{C} 8 \mathrm{D}$ & $111.3(4)$ \\
\hline $\mathrm{C} 7 \mathrm{~B}-\mathrm{C} 8 \mathrm{~B}-\mathrm{C} 9 \mathrm{~B}$ & $110.2(4)$ & $\mathrm{N} 2 \mathrm{D}-\mathrm{C} 10 \mathrm{D}-\mathrm{C} 11 \mathrm{D}$ & $110.2(4)$ \\
\hline $\mathrm{C} 10 \mathrm{~B}-\mathrm{C} 8 \mathrm{~B}-\mathrm{C} 9 \mathrm{~B}$ & $114.2(4)$ & $\mathrm{C} 8 \mathrm{D}-\mathrm{C} 10 \mathrm{D}-\mathrm{C} 11 \mathrm{D}$ & $112.3(4)$ \\
\hline $\mathrm{C} 7 \mathrm{~B}-\mathrm{C} 8 \mathrm{~B}-\mathrm{H} 8 \mathrm{C}$ & 106.9 & $\mathrm{~N} 2 \mathrm{D}-\mathrm{C} 10 \mathrm{D}-\mathrm{H} 10 \mathrm{G}$ & 107.6 \\
\hline $\mathrm{C} 10 \mathrm{~B}-\mathrm{C} 8 \mathrm{~B}-\mathrm{H} 8 \mathrm{C}$ & 106.9 & $\mathrm{C} 8 \mathrm{D}-\mathrm{C} 10 \mathrm{D}-\mathrm{H} 10 \mathrm{G}$ & 107.6 \\
\hline $\mathrm{C} 9 \mathrm{~B}-\mathrm{C} 8 \mathrm{~B}-\mathrm{H} 8 \mathrm{C}$ & 106.9 & $\mathrm{C} 11 \mathrm{D}-\mathrm{C} 10 \mathrm{D}-\mathrm{H} 10 \mathrm{G}$ & 107.6 \\
\hline $\mathrm{N} 1 \mathrm{~B}-\mathrm{C} 9 \mathrm{~B}-\mathrm{C} 8 \mathrm{~B}$ & $114.6(4)$ & $\mathrm{C} 12 \mathrm{D}-\mathrm{C} 11 \mathrm{D}-\mathrm{C} 10 \mathrm{D}$ & $112.9(5)$ \\
\hline $\mathrm{N} 1 \mathrm{~B}-\mathrm{C} 9 \mathrm{~B}-\mathrm{H} 9 \mathrm{C}$ & 108.6 & $\mathrm{C} 12 \mathrm{D}-\mathrm{C} 11 \mathrm{D}-\mathrm{H} 11 \mathrm{G}$ & 109.0 \\
\hline $\mathrm{C} 8 \mathrm{~B}-\mathrm{C} 9 \mathrm{~B}-\mathrm{H} 9 \mathrm{C}$ & 108.6 & $\mathrm{C} 10 \mathrm{D}-\mathrm{C} 11 \mathrm{D}-\mathrm{H} 11 \mathrm{G}$ & 109.0 \\
\hline $\mathrm{N} 1 \mathrm{~B}-\mathrm{C} 9 \mathrm{~B}-\mathrm{H} 9 \mathrm{D}$ & 108.6 & $\mathrm{C} 12 \mathrm{D}-\mathrm{C} 11 \mathrm{D}-\mathrm{H} 11 \mathrm{H}$ & 109.0 \\
\hline $\mathrm{C} 8 \mathrm{~B}-\mathrm{C} 9 \mathrm{~B}-\mathrm{H} 9 \mathrm{D}$ & 108.6 & $\mathrm{C} 10 \mathrm{D}-\mathrm{C} 11 \mathrm{D}-\mathrm{H} 11 \mathrm{H}$ & 109.0 \\
\hline $\mathrm{H} 9 \mathrm{C}-\mathrm{C} 9 \mathrm{~B}-\mathrm{H} 9 \mathrm{D}$ & 107.6 & $\mathrm{H} 11 \mathrm{G}-\mathrm{C} 11 \mathrm{D}-\mathrm{H} 11 \mathrm{H}$ & 107.8 \\
\hline $\mathrm{N} 2 \mathrm{~B}-\mathrm{C} 10 \mathrm{~B}-\mathrm{C} 8 \mathrm{~B}$ & $109.5(4)$ & $\mathrm{C} 13 \mathrm{D}-\mathrm{C} 12 \mathrm{D}-\mathrm{C} 11 \mathrm{D}$ & $109.8(5)$ \\
\hline $\mathrm{N} 2 \mathrm{~B}-\mathrm{C} 10 \mathrm{~B}-\mathrm{C} 11 \mathrm{~B}$ & $110.0(4)$ & $\mathrm{C} 13 \mathrm{D}-\mathrm{C} 12 \mathrm{D}-\mathrm{H} 12 \mathrm{G}$ & 109.7 \\
\hline $\mathrm{C} 8 \mathrm{~B}-\mathrm{C} 10 \mathrm{~B}-\mathrm{C} 11 \mathrm{~B}$ & $113.2(4)$ & $\mathrm{C} 11 \mathrm{D}-\mathrm{C} 12 \mathrm{D}-\mathrm{H} 12 \mathrm{G}$ & 109.7 \\
\hline $\mathrm{N} 2 \mathrm{~B}-\mathrm{C} 10 \mathrm{~B}-\mathrm{H} 10 \mathrm{C}$ & 108.0 & $\mathrm{C} 13 \mathrm{D}-\mathrm{C} 12 \mathrm{D}-\mathrm{H} 12 \mathrm{H}$ & 109.7 \\
\hline $\mathrm{C} 8 \mathrm{~B}-\mathrm{C} 10 \mathrm{~B}-\mathrm{H} 10 \mathrm{C}$ & 108.0 & $\mathrm{C} 11 \mathrm{D}-\mathrm{C} 12 \mathrm{D}-\mathrm{H} 12 \mathrm{H}$ & 109.7 \\
\hline $\mathrm{C} 11 \mathrm{~B}-\mathrm{C} 10 \mathrm{~B}-\mathrm{H} 10 \mathrm{C}$ & 108.0 & $\mathrm{H} 12 \mathrm{G}-\mathrm{C} 12 \mathrm{D}-\mathrm{H} 12 \mathrm{H}$ & 108.2 \\
\hline $\mathrm{C} 12 \mathrm{~B}-\mathrm{C} 11 \mathrm{~B}-\mathrm{C} 10 \mathrm{~B}$ & $113.0(5)$ & $\mathrm{C} 12 \mathrm{D}-\mathrm{C} 13 \mathrm{D}-\mathrm{C} 14 \mathrm{D}$ & $110.7(5)$ \\
\hline $\mathrm{C} 12 \mathrm{~B}-\mathrm{C} 11 \mathrm{~B}-\mathrm{H} 11 \mathrm{C}$ & 109.0 & $\mathrm{C} 12 \mathrm{D}-\mathrm{C} 13 \mathrm{D}-\mathrm{H} 13 \mathrm{G}$ & 109.5 \\
\hline $\mathrm{C} 10 \mathrm{~B}-\mathrm{C} 11 \mathrm{~B}-\mathrm{H} 11 \mathrm{C}$ & 109.0 & $\mathrm{C} 14 \mathrm{D}-\mathrm{C} 13 \mathrm{D}-\mathrm{H} 13 \mathrm{G}$ & 109.5 \\
\hline $\mathrm{C} 12 \mathrm{~B}-\mathrm{C} 11 \mathrm{~B}-\mathrm{H} 11 \mathrm{D}$ & 109.0 & $\mathrm{C} 12 \mathrm{D}-\mathrm{C} 13 \mathrm{D}-\mathrm{H} 13 \mathrm{H}$ & 109.5 \\
\hline $\mathrm{C} 10 \mathrm{~B}-\mathrm{C} 11 \mathrm{~B}-\mathrm{H} 11 \mathrm{D}$ & 109.0 & $\mathrm{C} 14 \mathrm{D}-\mathrm{C} 13 \mathrm{D}-\mathrm{H} 13 \mathrm{H}$ & 109.5 \\
\hline
\end{tabular}




$\begin{array}{ll}\text { H11C-C11B-H11D } & 107.8 \\ \text { C13B-C12B-C11B } & 111.3(5) \\ \text { C13B-C12B-H12C } & 109.4 \\ \text { C11B-C12B-H12C } & 109.4 \\ \text { C13B-C12B-H12D } & 109.4 \\ \text { C11B-C12B-H12D } & 109.4 \\ \text { H12C-C12B-H12D } & 108.0 \\ \text { C12B-C13B-C14B } & 110.2(5) \\ \text { C12B-C13B-H13C } & 109.6 \\ \text { C14B-C13B-H13C } & 109.6 \\ \text { C12B-C13B-H13D } & 109.6 \\ \text { C14B-C13B-H13D } & 109.6 \\ \text { H13C-C13B-H13D } & 108.1 \\ \text { C13B-C14B-N2B } & 111.8(4) \\ \text { C13B-C14B-H14C } & 109.3 \\ \text { N2B-C14B-H14C } & 109.3 \\ \text { C13B-C14B-H14D } & 109.3 \\ \text { N2B-C14B-H14D } & 109.3 \\ \text { H14C-C14B-H14D } & 107.9 \\ \text { N2B-C15B-C6B } & 112.2(4) \\ \text { N2B-C15B-H15C } & 109.2 \\ \text { C6B-C15B-H15C } & 109.2 \\ \text { N2B-C15B-H15D } & 109.2 \\ \text { C6B-C15B-H15D } & 109.2 \\ \text { H15C-C15B-H15D } & 107.9 \\ \text { C5C-N1C-C1C } & 122.0(4) \\ \text { C5C-N1C-C9C } & 122.7(4) \\ \text { C1C-N1C-C9C } & 115.2(4) \\ \text { C15C-N2C-C14C } & 112.6(4) \\ \text { C15C-N2C-C10C } & 113.5(4) \\ \text { C14C-N2C-C10C } & 111.6(4) \\ \text { C15C-N2C-H2EN } & 105(6) \\ \text { C14C-N2C-H2EN } & 111(6) \\ \text { C10C-N2C-H2EN } & 103(6) \\ \text { O1C-C1C-N1C } & 119.0(4) \\ \text { O1C-C1C-C2C } & 124.9(4) \\ \text { N1C-C1C-C2C } & 116.1(4) \\ & \end{array}$

\begin{tabular}{|c|c|}
\hline $\mathrm{H} 13 \mathrm{G}-\mathrm{C} 13 \mathrm{D}-\mathrm{H} 13 \mathrm{H}$ & 108.1 \\
\hline $\mathrm{N} 2 \mathrm{D}-\mathrm{C} 14 \mathrm{D}-\mathrm{C} 13 \mathrm{D}$ & $111.0(4)$ \\
\hline $\mathrm{N} 2 \mathrm{D}-\mathrm{C} 14 \mathrm{D}-\mathrm{H} 14 \mathrm{G}$ & 109.4 \\
\hline $\mathrm{C} 13 \mathrm{D}-\mathrm{C} 14 \mathrm{D}-\mathrm{H} 14 \mathrm{G}$ & 109.4 \\
\hline $\mathrm{N} 2 \mathrm{D}-\mathrm{C} 14 \mathrm{D}-\mathrm{H} 14 \mathrm{H}$ & 109.4 \\
\hline $\mathrm{C} 13 \mathrm{D}-\mathrm{C} 14 \mathrm{D}-\mathrm{H} 14 \mathrm{H}$ & 109.4 \\
\hline $\mathrm{H} 14 \mathrm{G}-\mathrm{C} 14 \mathrm{D}-\mathrm{H} 14 \mathrm{H}$ & 108.0 \\
\hline $\mathrm{N} 2 \mathrm{D}-\mathrm{C} 15 \mathrm{D}-\mathrm{C} 6 \mathrm{D}$ & $111.7(4)$ \\
\hline $\mathrm{N} 2 \mathrm{D}-\mathrm{C} 15 \mathrm{D}-\mathrm{H} 15 \mathrm{G}$ & 109.3 \\
\hline $\mathrm{C} 6 \mathrm{D}-\mathrm{C} 15 \mathrm{D}-\mathrm{H} 15 \mathrm{G}$ & 109.3 \\
\hline $\mathrm{N} 2 \mathrm{D}-\mathrm{C} 15 \mathrm{D}-\mathrm{H} 15 \mathrm{H}$ & 109.3 \\
\hline $\mathrm{C} 6 \mathrm{D}-\mathrm{C} 15 \mathrm{D}-\mathrm{H} 15 \mathrm{H}$ & 109.3 \\
\hline $\mathrm{H} 15 \mathrm{G}-\mathrm{C} 15 \mathrm{D}-\mathrm{H} 15 \mathrm{H}$ & 107.9 \\
\hline $\mathrm{O} 13-\mathrm{Cl1}-\mathrm{O} 14$ & $112.3(5)$ \\
\hline $\mathrm{O} 13-\mathrm{C} 11-\mathrm{O} 11$ & $112.0(5)$ \\
\hline $\mathrm{O} 14-\mathrm{C} 11-\mathrm{O} 11$ & $111.1(6)$ \\
\hline $\mathrm{O} 13-\mathrm{Cl1}-\mathrm{O} 12$ & $110.8(6)$ \\
\hline $\mathrm{O} 14-\mathrm{C} 11-\mathrm{O} 12$ & $106.1(7)$ \\
\hline $\mathrm{O} 11-\mathrm{C} 11-\mathrm{O} 12$ & $104.1(7)$ \\
\hline $\mathrm{O} 23-\mathrm{C} 2-\mathrm{O} 21$ & $100.5(13)$ \\
\hline $\mathrm{O} 23-\mathrm{Cl} 2-\mathrm{O} 22$ & $109.0(11)$ \\
\hline $\mathrm{O} 21-\mathrm{Cl} 2-\mathrm{O} 22$ & $108.4(11)$ \\
\hline $\mathrm{O} 23-\mathrm{Cl} 2-\mathrm{O} 24$ & $112.3(6)$ \\
\hline $\mathrm{O} 21-\mathrm{Cl} 2-\mathrm{O} 24$ & $112.1(8)$ \\
\hline $\mathrm{O} 22-\mathrm{C} 2-\mathrm{O} 24$ & $113.6(5)$ \\
\hline $\mathrm{O} 31-\mathrm{Cl} 3-\mathrm{O} 32$ & $107.0(7)$ \\
\hline $\mathrm{O} 31-\mathrm{Cl} 3-\mathrm{O} 33$ & $112.1(5)$ \\
\hline $\mathrm{O} 32-\mathrm{Cl} 3-\mathrm{O} 33$ & $110.5(6)$ \\
\hline $\mathrm{O} 31-\mathrm{Cl} 3-\mathrm{O} 34$ & $110.8(7)$ \\
\hline $\mathrm{O} 32-\mathrm{Cl} 3-\mathrm{O} 34$ & $105.4(8)$ \\
\hline $\mathrm{O} 33-\mathrm{Cl} 3-\mathrm{O} 34$ & $110.8(4)$ \\
\hline $\mathrm{O} 42-\mathrm{Cl} 4-\mathrm{O} 43$ & $113.4(5)$ \\
\hline $\mathrm{O} 42-\mathrm{Cl} 4-\mathrm{O} 41$ & $102.8(8)$ \\
\hline $\mathrm{O} 43-\mathrm{Cl} 4-\mathrm{O} 41$ & $105.1(6)$ \\
\hline $\mathrm{O} 42-\mathrm{Cl} 4-\mathrm{O} 44$ & $115.1(6)$ \\
\hline $\mathrm{O} 43-\mathrm{Cl} 4-\mathrm{O} 44$ & $113.9(3)$ \\
\hline $\mathrm{O} 41-\mathrm{Cl} 4-\mathrm{O} 44$ & $105.0(6)$ \\
\hline
\end{tabular}

Hydrogen-bond geometry $\left(\AA,{ }^{\circ}\right)$

\begin{tabular}{lllll}
\hline$D-\mathrm{H} \cdots A$ & $D-\mathrm{H}$ & $\mathrm{H} \cdots A$ & $D \cdots A$ & $D-\mathrm{H} \cdots A$ \\
\hline $\mathrm{N} 2 A-\mathrm{H} 2 A N \cdots \mathrm{O} 1 B$ & $1.03(5)$ & $1.91(6)$ & $2.741(6)$ & $136(5)$ \\
$\mathrm{N} 2 B-\mathrm{H} 2 C N \cdots \mathrm{O} 1 A$ & $0.77(7)$ & $2.00(6)$ & $2.742(5)$ & $163(6)$ \\
$\mathrm{N} 2 C-\mathrm{H} 2 E N \cdots \mathrm{O} 1 D^{\mathrm{i}}$ & $0.90(9)$ & $2.00(9)$ & $2.735(6)$ & $138(8)$ \\
$\mathrm{N} 2 D-\mathrm{H} 2 G N \cdots \mathrm{O} 1 C^{\mathrm{ii}}$ & $1.05(5)$ & $1.74(5)$ & $2.754(5)$ & $159(5)$
\end{tabular}

Symmetry codes: (i) $x+1, y, z$; (ii) $x-1, y, z$. 